\title{
Second order mem-circuits
}

\author{
RICARDO RIAZA
}

\begin{abstract}
This paper presents a comprehensive taxonomy of so-called second order memory devices, which include charge-controlled memcapacitors and flux-controlled meminductors, among other novel circuit elements. These devices, which are classified according to their differential and state orders, are necessary to get a complete extension of the family of classical nonlinear circuit elements (resistors, capacitors, inductors) for all possible controlling variables. Using a fully nonlinear formalism, we obtain nondegeneracy conditions for a broad class of second order mem-circuits. This class of circuits is expected to yield a rich dynamic behavior; in this regard we explore certain bifurcation phenomena exhibited by a family of circuits including a charge-controlled memcapacitor and a flux-controlled meminductor, providing some directions for future research.
\end{abstract}

Keywords: nonlinear circuit, memristor, memcapacitor, meminductor, state-space model, bifurcation, differential-algebraic equation.

\section{Introduction}

The announcement in 2008 of a nanoscale device with a memristive characteristic [1] has had a great impact in electronic engineering. Applications of memristors and other memory devices are being reported in many fields: see [2]-[16] and references therein. Since the seminal work of Chua [17], the memristor has led to a revision of the analytical foundations of circuit theory in several directions.

One of these directions concerns the nature of the variables involved in nonlinear circuit models. In this regard, higher order devices are those which do not admit a description in terms of the fundamental circuit variables $q, \varphi, i, v[14]$. Devices such as charge-controlled memcapacitors and flux-controlled meminductors [5], or the $\sigma$ - $\rho$ devices proposed in [3], involve new variables, namely the integral variables $\sigma=\int q=\iint i$ or $\rho=\int \varphi=\iint v$, which are located beyond the classical limits of circuit theory (throughout the document we use the notation $y=\int x$ and $z=\iint x$ as abbreviations for $\left.y(t)=\int_{-\infty}^{t} x(s) d s, z(t)=\int_{-\infty}^{t}\left(\int_{-\infty}^{s} x(\tau) d \tau\right) d s\right)$.

The first purpose of the present paper is to extend the results discussed in [14] to accommodate these and other related devices in a systematic framework. In particular, we will clarify the variables that one can eliminate in the description of such devices; for instance, the description of a charge-controlled memcapacitor necessarily involves $q$ but also the second order (that is, 
involving a second integral) variable $\sigma$, besides the voltage $v$, in contrast to a voltage-controlled memcapacitor which can be described in terms of the first order variables $q, \varphi$ (again, together with $v$ ). Find details in Sections 2 and 3. For better readability, the ideas discussed in these and later Sections are illustrated, as soon as they are introduced, by means of a running circuit example which is presented at the end of this Introduction.

Charge-controlled memcapacitors and flux-controlled meminductors, together with voltagecontrolled memcapacitors, current-controlled meminductors and charge- and flux-controlled memristors, provide a complete extension of the classical nonlinear devices, namely, charge- and voltage-controlled capacitors, flux- and current-controlled inductors, and current- and voltagecontrolled resistors. For this reason, this set of devices deserves special attention; in Section 4 we present non-degeneracy conditions which guarantee the existence of a state-space description for mem-circuits (namely, circuits including elements with memory such as memristors, memcapacitors or meminductors) based on these building blocks.

The second purpose of the present paper is to provide some insight into the rich dynamical behavior which may be displayed by second order mem-circuits. In this direction, we analyze in Section 5 several dynamical properties of a family of circuits including a charge-controlled memcapacitor and a flux-controlled meminductor, addressing some qualitative changes which may result from the failure of the assumptions that support the aforementioned non-degeneracy analysis. This section is also aimed at motivating further research. Finally, some concluding remarks can be found in Section 6, and several auxiliary mathematical notions and results are compiled in the Appendix.

\section{A running example}

We will use the circuit depicted in Figure 1 to illustrate the notions and ideas discussed throughout the paper. This circuit is defined by two nonlinear oscillators coupled by a memcapacitor $\left(C_{12}\right.$ in the Figure), driven by a voltage source $V_{12}$. The two oscillators $(i=1,2)$ are defined by the series connection of a voltage source $V_{i}$, with a parasitic resistance $R_{i}$, a linear capacitor with capacitance $C_{i}$, and a Josephson junction; we model the latter by a parallel connection of a nonlinear inductor ( $L_{i}$ in the figure) and a $\varphi$-memristor with memductance $W_{i}$, as in [18]. This model accommodates parasitic effects of memristive nature in the Josephson junction; to simplify matters, we neglect other capacitive and resistive effects in the junction. The use of a memcapacitor to couple such oscillators has been proposed in quantum computation [13].

\section{The differential and the state order of a device}

Extending the results of [14], we discuss below the notions of the differential and the state order of a circuit element. We assume for the sake of simplicity that no more than the variables $\sigma, \rho$, $q, \varphi, i, v$ (besides the time $t$ in time-varying cases and/or in independent sources) are involved in the device description; to the knowledge of the author, no physical device involving the third integrals $\iiint i$ or $\iiint v$ has been reported in the literature. 


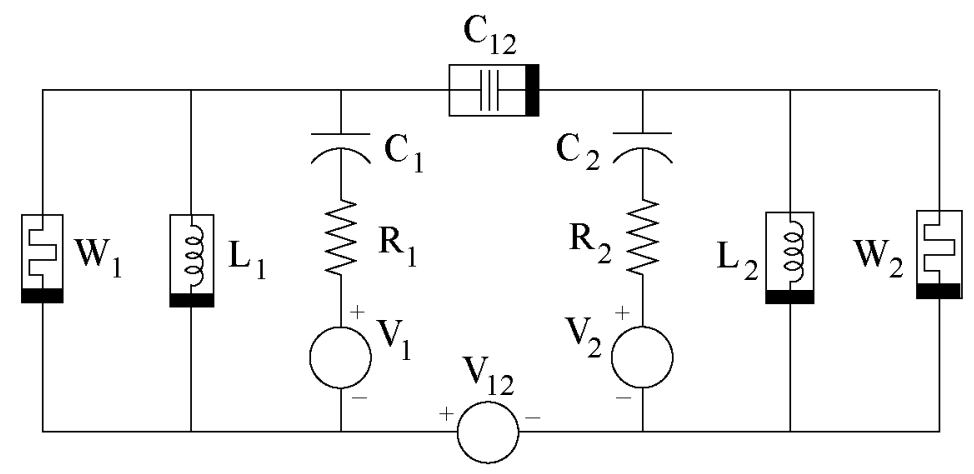

Figure 1: Example.

A preliminary discussion is necessary. Note that several different descriptions are possible for a given device; for instance, we may describe Chua's charge-controlled memristor [17] either by means of its flux-charge relation

$$
\varphi=\phi(q)
$$

or, differentiating (1), by

$$
v=M(q) i
$$

where $M(q)=\frac{d \phi(q)}{d q}$ is the so-called memristance. Note that (2), contrary to (1), reflects explicitly the memory effect of the memristor, which relies on the dependence of $M$ on the variable $q=\int i$, this way capturing the device history. Another obvious advantage of (2) over (1) is that there is no reason to keep track of both the flux and the charge as dynamic variables since their values are constrained by the relation (1).

Provided that the characteristic of a device is smooth enough (details are given below), such a differentiation process can always be carried out until $i$ and/or $v$ appear explicitly. If in such a description $\sigma$ and/or $\rho$ are present, the device is said to have differential order two; if they are not, but $q$ and/or $\varphi$ appear explicitly, then the device is said to have differential order one. If the description only involves $i$ and/or $v$, the device is said to have differential order zero. In other words, the differential order is the highest order of an integral involved in a device description in which $i$ and/or $v$ appear explicitly; this will be the order of the differential equation associated with the device (e.g. in a device with differential order two, the dynamical description would carry at least one of the identities $\sigma^{\prime \prime}=i, \rho^{\prime \prime}=v$; throughout the document we use the prime ${ }^{\prime}$ to denote differentiation with respect to time, and drop the argument $t$ for ease of notation). The term "order" alone will also be used to refer to the differential order. The (differential) order of a circuit is the highest order of its devices. Regarding the smoothness of the description, in order to carry out the necessary derivations for $i$ and/or $v$ to appear explicitly, for a device with differential order $n$ it is enough to assume in general that partial derivatives exist up to order $n$; in many cases this assumption may be relaxed.

In order to get a better understanding of this notion, think of a hypothetical "linear memristor" governed e.g. by a relation $\varphi=R q$ with constant $R$. This also admits, by differentiation, the description $v=R i$, so that the device amounts to a linear resistor. In our setting, such a "linear memristor" (which for these reasons is a contradiction in terms) would not be an order 
one but an order zero device (i.e. a resistor) because it admits a description just in terms of $i$ and $v$.

Again in terms of a description involving $i$ and/or $v$, the state order of a device is the number of dynamic variables (namely, $\sigma, \rho, q, \varphi$ ) involved in such a description. This notion captures the number of degrees of dynamic freedom that the device introduces (alternatively, the number of initial conditions that can be arbitrarily given). Resistors, as well as voltage and current sources, have state order zero. In terms of the example above, the memristor has state order one, and this means that every memristor introduces one (not two) degree(s) of dynamic freedom, associated with the charge $q$ (cf. $[17,19]$ ); by describing the device characteristic via (2) we discard the flux $\varphi$. Notice that, given an initial condition $q(0)$, we cannot give an arbitrary value to $\varphi(0)$ since the latter must be given by $\phi(q(0))$.

Devices with order zero and one. According to the notions discussed above, devices with differential order zero are (possibly nonlinear) resistors as well as voltage and current sources, since they only involve the variables $i$ and/or $v$ (besides an explicit time dependence in independent sources). The key aspect is that all these devices have descriptions which do not involve either $q, \varphi$ or higher order variables such as $\sigma$ or $\rho$.

First order circuit devices are those defined by a relation among some of the fundamental variables $q, \varphi, i, v$ in which $q$ and/or $\varphi$ appear explicitly. Capacitors and inductors, and also memristors, are first order devices. Regarding memristors, as detailed in [14] (2) can be seen as a particular instance of the fully nonlinear characteristic $v=\eta(q, i)$ which defines the so-called $q$-memristors. The incremental memristance is $\frac{\partial \eta(q, i)}{\partial i}$. The dual relation for $\varphi$-memristors has the form $i=\zeta(\varphi, v)$, the incremental memductance being $\frac{\partial \zeta(\varphi, v)}{\partial v}$; in particular, Chua's fluxcontrolled memristors can be described either by a map $q=\xi(\varphi)$ or, better, in the differentiated form $i=W(\varphi) v$ in terms of the memductance $W(\varphi)=\frac{d \xi(\varphi)}{d \varphi}$, thus eliminating the charge $q$. The zero-crossing property of Chua's memristors read, in a fully nonlinear setting, as $\eta(q, 0)=0$ or $\zeta(\varphi, 0)=0$, respectively. Capacitors, inductors and memristors have state order one.

As detailed in subsection 3.1 below, voltage-controlled memcapacitors and current-controlled meminductors are also first order devices, since in general their characteristics can be written in the form $q=\omega(\varphi, v)$ or $\varphi=\theta(q, i)$; the zero-crossing property read for them $\omega(\varphi, 0)=0$ and $\theta(q, 0)=0$, respectively. These devices have state order two since their characteristics involve both $q$ and $\varphi$.

\section{$3 \quad$ Second order circuit devices}

\subsection{Second order devices}

Beyond the first order setting, higher order devices involve additional variables such as the second integrals $\sigma, \rho$ of the branch current and voltage. With the use of these additional variables we may accommodate e.g. the charge-controlled memcapacitors or flux-controlled meminductors of [5] and their fully nonlinear counterparts described later. Second order devices are the focus of this paper and for this reason we extract and emphasize this particular case from the general 
framework of Section 2.

Definition 1. A second order description for a circuit device is a differentiable description of the form

$$
h(\sigma, \rho, q, \varphi, i, v, t)=0,
$$

where at least one of the partial derivatives $\frac{\partial h}{\partial \sigma}, \frac{\partial h}{\partial \rho}$ does not vanish identically.

To carry out differentiations, in specific cases (in particular in the $\sigma-\rho$ devices discussed later) we will need to assume that $h$ is twice differentiable.

Definition 2. A second order device is a circuit element which admits a second order description, but not a first order one.

As discussed in Section 2, ruling out first order descriptions in Definition 2 is necessary for the sake of consistency. For instance, voltage-controlled memcapacitors and current-controlled meminductors, as introduced in [5], are (in our terms) defined by second order descriptions $\sigma=\mu(\varphi)$ and $\rho=\kappa(q)$. However, using $\sigma^{\prime}=q, \rho^{\prime}=\varphi$ by differentiation we discard $\sigma$ and $\rho$ in the corresponding first order descriptions $q=C(\varphi) v$ and $\varphi=L(q) i$. These descriptions only involve (some of) the first order variables $q, \varphi, i$ and $v$ and the devices are therefore first order ones. This is also the case for their fully nonlinear counterparts, defined by $q=\omega(\varphi, v)$ and $\varphi=\theta(q, i)[14]$. Things will be different for charge-controlled memcapacitors and flux-controlled meminductors, for which there is no chance to eliminate a second order integral variable ( $\sigma$ or $\rho$, respectively).

Charge-controlled memcapacitors. A charge-controlled memcapacitor is a second order device defined by a characteristic of the form

$$
v=\nu(\sigma, q, t)
$$

where $\nu$ is a differentiable map for which neither of the derivatives $\frac{\partial \nu}{\partial \sigma}, \frac{\partial \nu}{\partial q}$ vanishes identically. In particular, when $\nu$ is time-invariant and linear in $q$, one gets the device discussed by Di Ventra et al. in [5], for which the characteristic (4) reads as $v=E(\sigma) q$ and arises as the differentiated form of a relation $\varphi=\alpha(\sigma)$. Here $E$ is the inverse memcapacitance (or elastance), which introduces memory effects in the circuit because of its dependence on $\sigma$. In general, the zero-crossing property reads as $\nu(\sigma, 0, t)=0$, and the incremental inverse memcapacitance $E(\sigma, q, t)$ is defined by the partial derivative $\frac{\partial \nu(\sigma, q, t)}{\partial q}$. Notice that when $\nu$ is time-invariant and linear in $q$, we have $\frac{\partial \nu(\sigma, q)}{\partial q}=E(\sigma)=\frac{d \alpha(\sigma)}{d \sigma}$.

Flux-controlled meminductors. The dual device is a flux-controlled meminductor, which is a second order device governed by the characteristic

$$
i=\chi(\rho, \varphi, t)
$$

where $\chi$ is a differentiable map such that neither of the derivatives $\frac{\partial \chi}{\partial \rho}$, $\frac{\partial \chi}{\partial \varphi}$ vanishes identically. The zero-crossing property is now $\chi(\rho, 0, t)=0$. If $\chi$ is time-invariant and linear in $\varphi$, we get 
again the corresponding device discussed in [5], with a characteristic $i=\mathcal{R}(\rho) \varphi$ which is the differentiated form of $q=\beta(\rho)$. Here $\mathcal{R}$ is the inverse meminductance (or reluctance). In the fully nonlinear case, the incremental inverse meminductance is $\mathcal{R}(\rho, \varphi, t)=\frac{\partial \chi(\rho, \varphi, t)}{\partial \varphi}$.

From the descriptions (4) and (5) it follows that charge-controlled memcapacitors and fluxcontrolled meminductors have state order two, namely, their behavior can be described in terms of two dynamic variables; note that neither $\rho$ nor $\varphi$ (resp. $\sigma, q$ ) arise in the characteristic (4) (resp. (5)). Note that voltage-controlled memcapacitors and current-controlled meminductors also have state order two.

$\sigma-\rho$ devices. Other second order devices have been discussed in the literature. For symmetry reasons, the existence of devices directly relating the second order variables $\sigma$ and $\rho$ has been conjectured in [3]. Focus, without loss of generality, on the $\sigma$-controlled characteristic $\rho=f(\sigma)$. It is natural to wonder how many independent dynamic variables (out of $\sigma, \rho, q, \varphi$ ) are actually involved in this device or, in other terms, what is its state order. Provided that $f$ is twice differentiable, one differentiation yields $\varphi=\frac{d f(\sigma)}{d \sigma} q$. This way we eliminate the second order variable $\rho$ from the device description, but note that $\sigma$ is still present; in other words, this is also a second order description. Another differentiation step leads to

$$
v=\frac{d^{2} f(\sigma)}{d \sigma^{2}} q^{2}+\frac{d f(\sigma)}{d \sigma} i
$$

which is also a second order device description in which $\varphi$ is no longer present. In light of (6), both the differential and the state order of the device are two.

By writing (6) as $v=v_{1}+v_{2}=\frac{d^{2} f(\sigma)}{d \sigma^{2}} q^{2}+\frac{d f(\sigma)}{d \sigma} i$, it is worth noting that the device combines two memory effects. First, there is a nonlinear memcapacitive effect described by $v_{1}=\frac{d^{2} f(\sigma)}{d \sigma^{2}} q^{2}$ (cf. (4)). The second term, $v_{2}=\frac{d f(\sigma)}{d \sigma} i$, may be understood to define a memristive effect, since this term directly relates voltage and current; in this case the memory effect does not rely on $q$, as in (2), but on the second order variable $\sigma$.

\subsection{Classical vs. memory devices}

The fully nonlinear memristors, memcapacitors and meminductors discussed above provide an extension of the six types of classical nonlinear devices, as detailed below. For notational simplicity we assume all devices to be time-invariant.

1. A current-controlled resistor, defined by a characteristic $v=\gamma_{1}(i)$, can be seen as a memoryless case of a $q$-memristor $v=\eta(q, i)$ in which there is no dependence on $q$.

2. A voltage-controlled resistor, governed by a relation of the form $i=\gamma_{2}(v)$, is an instance without memory of a $\varphi$-memristor $i=\zeta(\varphi, v)$ when there is no dependence on $\varphi$.

3. A voltage-controlled capacitor, for which $q=\gamma_{3}(v)$, is in turn a memoryless version of a voltage-controlled memcapacitor $q=\omega(\varphi, v)$.

4. A charge-controlled capacitor, defined by a mapping of the form $v=\gamma_{4}(q)$, is a memoryless version of a charge-controlled memcapacitor $v=\nu(\sigma, q)$. 
5. A current-controlled inductor, governed by $\varphi=\gamma_{5}(i)$, generalizes to a current-controlled meminductor $\varphi=\theta(q, i)$.

6. Finally, a flux-controlled inductor, characterized by a map $i=\gamma_{6}(\varphi)$, is a memoryless version of a flux-controlled meminductor $i=\chi(\rho, \varphi)$.

Note that all the memory devices involve one additional dynamic variable with respect to their memoryless counterparts, namely $q$ or $\varphi$ for memristors, $\varphi$ or $\sigma$ for memcapacitors, $q$ or $\rho$ for meminductors. In other terms, the state order increases by one in all cases when going from the classical (memoryless) setting to the memory-based one. This additional variable is responsible for the memory effect in items 1,2,3 and 5 above; in the cases 4 and 6 , both $\sigma$ and $q$, or $\rho$ and $\varphi$, may incorporate the memory effect.

\section{Non-degeneracy}

The taxonomy presented in subsection 3.2 makes it worth analyzing in more detail the family of circuits defined by the devices listed in items 1-6. We present in Theorem 1 (subsection 4.5) non-degeneracy conditions for such circuits. We say that a nonlinear circuit is non-degenerate if the set of trajectories defines a manifold whose dimension (the so-called state dimension) equals the sum of the state orders of its devices. We illustrate the different notions and results by means of the running example presented at the end of the Introduction.

\subsection{Topological degeneracies}

The non-degeneracy notion involves a two-fold requirement; the first one is that the circuit displays no topological degeneracies, which would drive the state dimension below the sum of state orders since they restrict the admissible values of voltage drops in capacitors/memcapacitors or the currents in inductors/meminductors. In this context, topological degeneracies are loops formed by voltage sources, capacitors and/or memcapacitors (find an example in Figure 2, obtained after short-circuiting $R_{1}$ and $R_{2}$ in Figure 1), and cutsets defined by current sources, inductors and/or meminductors (a simple example defined by a current source and an inductor is depicted in Figure 3(a)).

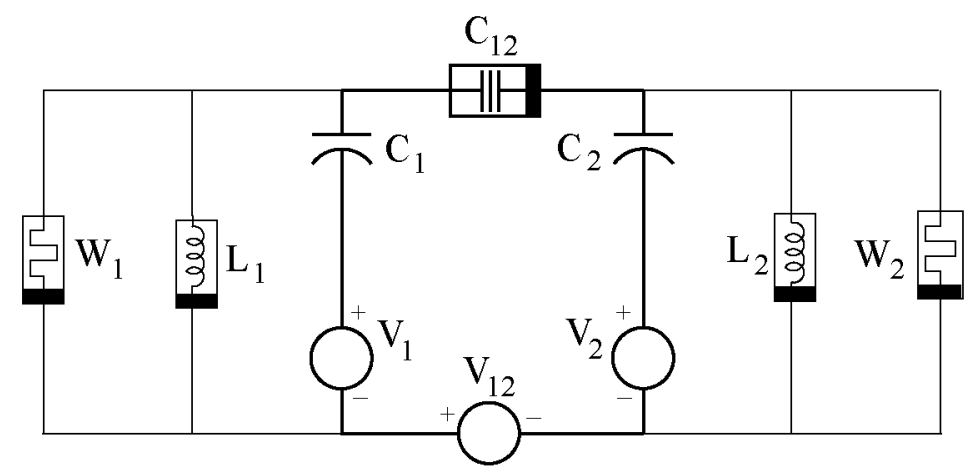

Figure 2: Short-circuiting $R_{1}$ and $R_{2}$ in Fig. 1 yields a topologically degenerate loop. 

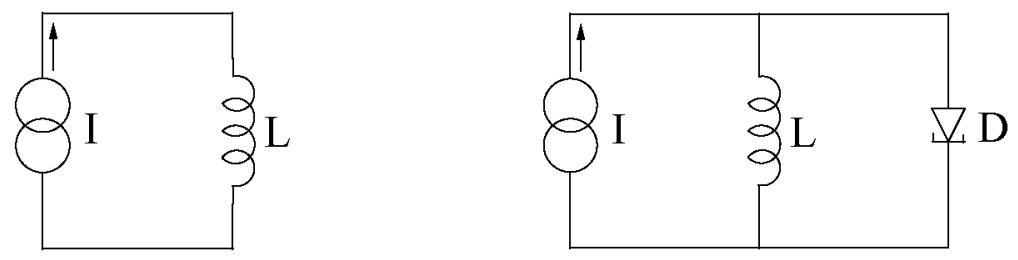

Figure 3: (a) IL-cutset (b) ILG-circuit with a tunnel diode.

The second requirement is that the devices' characteristics do not display singularities in order to guarantee that the dynamics is well-defined around any operating point; cf. [20, 21, 22] and references therein. A simple example of a singularity is obtained after adding a tunnel diode in parallel to the aforementioned IL-connection, as in Figure 3(b). The tunnel diode has a voltage-controlled characteristic $i=\gamma(v)$ and the important feature is that $\gamma^{\prime}(v)$ does vanish at two points $v_{1}, v_{2}$ (with $\gamma^{\prime}(v)<0$ for $v \in\left(v_{1}, v_{2}\right)$ ). By orienting the $L$ and $D$ branches top-down, letting $i_{l}$ and $v_{l}$ denote the current/voltage in the inductor branch, and writing the current through the diode as $I-i_{l}$, the equations modelling the circuit in Figure 3 (b) read as

$$
\begin{aligned}
L \frac{d i_{l}}{d t} & =v_{l} \\
0 & =I-i_{l}-\gamma\left(v_{l}\right) .
\end{aligned}
$$

Near the singular values $v_{l}=v_{1}$ or $v_{l}=v_{2}$, we cannot solve for $v_{l}$ in $(7 \mathrm{~b})$, since $v_{1}$ is a local maximum of $\gamma$ and therefore there is no local inverse of this function; the same happens around the local minimum at $v_{2}$. In circuit-theoretic terms, not even locally there is a chance to describe uniquely the voltage in terms of the current near the extrema of a tunnel diode characteristic. This leads to an impasse phenomenon which is well-studied in the circuit literature; find details in $[20,21,23]$.

\subsection{Devices}

For notational simplicity we will describe each one of the pair of devices in items 1-6 above by its memory version, in the understanding that e.g. the map $v_{m}=\eta_{m}\left(q_{m}, i_{m}\right)$ defines the characteristics of both current-controlled resistors and $q$-memristors; in the entries of $\eta_{m}$ corresponding to resistors there is simply no dependence on $q_{m}$. Analogously, $i_{w}=\zeta_{w}\left(\varphi_{w}, v_{w}\right)$ groups together voltage-controlled resistors and $\varphi$-memristors, $q_{m c}=\omega_{m c}\left(\varphi_{m c}, v_{m c}\right)$ describes voltage-controlled capacitors and memcapacitors, whereas $v_{m q}=\nu_{m q}\left(\sigma_{m q}, q_{m q}\right)$ describes charge-controlled capacitors and memcapacitors; $\varphi_{m l}=\theta_{m l}\left(q_{m l}, i_{m l}\right)$ groups together current-controlled inductors and meminductors, and $i_{m \varphi}=\chi_{m \varphi}\left(\rho_{m \varphi}, \varphi_{m \varphi}\right)$ describes both flux-controlled inductors and meminductors. Note that here and in the sequel all subscripts are meant to distinguish group of devices: $m$ corresponds to current-controlled resistors and $q$-memristors; $w$ stands for voltage-controlled resistors and $\varphi$-memristors; $m c$ represents voltage-controlled capacitors and memcapacitors; $m q$ denotes charge-controlled capacitors and memcapacitors; $m l$ corresponds to current-controlled 
inductors and meminductors; $m \varphi$ denotes flux-controlled inductors and meminductors; finally, we will use $u$ and $j$ as subscripts for voltage and current sources, so that $v_{u}, i_{u}$ are the voltage and the current in voltage source branches and $v_{j}, i_{j}$ are the voltage and the current in current source branches. It is also worth emphasizing that all variables and maps will in general be vector-valued (see e.g. (34) and (35) for the running example).

With this notation, we define the generalized incremental resistance, conductance, capacitance, elastance, inductance and reluctance matrices as the matrices of partial derivatives

$$
M=\frac{\partial \eta_{m}}{\partial i_{m}}, W=\frac{\partial \zeta_{w}}{\partial v_{w}}, C=\frac{\partial \omega_{m c}}{\partial v_{m c}}, E=\frac{\partial \nu_{m q}}{\partial q_{m q}}, L=\frac{\partial \theta_{m l}}{\partial i_{m l}}, \mathcal{R}=\frac{\partial \chi_{m \varphi}}{\partial \varphi_{m \varphi}} .
$$

Provided that there is no coupling effects among the memory and the memoryless version of each set of devices (e.g. among memcapacitors and capacitors), each one of these matrices amounts to a block-diagonal composition of two submatrices; for instance, $M=\operatorname{blockdiag}(\breve{M}, \hat{M})$, where $\breve{M}$ denotes the incremental resistance matrix for current-controlled resistors and $\hat{M}$ is the incremental memristance matrix for $q$-memristors. Note that $\hat{M}$ depends on $q_{m}$ but $\breve{M}$ does not. Analogously, the remaining matrices have the structure $W=\operatorname{blockdiag}(\breve{W}, \hat{W}), C=\operatorname{blockdiag}(\breve{C}, \hat{C})$, $E=\operatorname{blockdiag}(\check{E}, \hat{E}), L=\operatorname{blockdiag}(\check{L}, \hat{L})$ and $\mathcal{R}=\operatorname{blockdiag}(\check{\mathcal{R}}, \hat{\mathcal{R}})$.

The matrices $M$ and $W$ will be positive definite everywhere (cf. the Appendix). This positive definiteness assumption means that memristors and resistors are strictly locally passive. In turn, $C, E, L$ and $\mathcal{R}$ will be assumed to be invertible (and the corresponding devices termed nonsingular accordingly).

Devices in the running example. In the running example (Figure 1) we will assume that the memcapacitor is a charge-controlled one; the most general setting follows from the assumption that its characteristic has a fully nonlinear form

$$
v_{m q}=\nu_{m q}\left(\sigma_{m q}, q_{m q}\right)
$$

with $C_{12}=\left(\frac{\partial \nu_{m q}}{\partial q_{m q}}\right)^{-1}$. Note that the circuit only includes one memcapacitor and therefore $v_{m q}$ (resp. $i_{m q}$ ) will correspond to $v_{c_{12}}\left(\right.$ resp. $\left.i_{c_{12}}\right)$. The description of the device also involves the differential relations

$$
\begin{aligned}
\sigma_{m q}^{\prime} & =q_{m q} \\
q_{m q}^{\prime} & =i_{m q} .
\end{aligned}
$$

Both memristors are flux-controlled by relations of the form

$$
i_{w_{k}}=W_{k}\left(\varphi_{w_{k}}\right) v_{w_{k}}, k=1,2 .
$$

As detailed in [18], the memductance has the expression $W_{k}(\varphi)=W_{0} \cos \left(k_{0} \varphi\right)$ for certain constants $W_{0}, k_{0}$. The model must also take into account the differential equations

$$
\varphi_{w_{k}}^{\prime}=v_{w_{k}}, k=1,2 .
$$


The nonlinear inductors coming from the Josephson junctions (see e.g. [24]) are flux-controlled, with a characteristic of the form

$$
i_{l_{k}}=\chi_{k}\left(\varphi_{l_{k}}\right)=I_{0} \sin \left(c_{0} \varphi_{l_{k}}\right), \quad k=1,2,
$$

for certain physical constants $I_{0}, c_{0}$; with this notation we have $L_{k}=\left(\frac{\partial \chi_{k}}{\partial \varphi_{l_{k}}}\right)^{-1}$. The device description also includes the relations

$$
\varphi_{l_{k}}^{\prime}=v_{l_{k}}, \quad k=1,2
$$

Resistors and capacitors are linear, their characteristics just reading as

$$
v_{r_{k}}=R_{k} i_{r_{k}} \text { and } q_{c_{k}}=C_{k} v_{c_{k}}, k=1,2 .
$$

For capacitors we also need the differential relations

$$
q_{c_{k}}^{\prime}=i_{c_{k}}, k=1,2
$$

Finally, we will denote by $V_{1}(t), V_{2}(t)$ and $V_{12}(t)$ the voltage at the sources.

In later analyses we will mainly focus on strictly locally passive cases, in which the incremental memductances, resistances, capacitances and inductances are positive. We will also assume that the running example has no coupling effects. However, just for illustrative purposes regarding the form of the matrices introduced in (8) above, let us assume for a while that there is a capacitive coupling (with parameter $C_{\kappa}$ ) among the memoryless capacitors $C_{1}$ and $C_{2}$, but that none of them is coupled with the memcapacitor $C_{12}$. This would confer the matrix $C$ the structure

$$
C=\left(\begin{array}{ccc}
C_{1} & C_{\kappa} & 0 \\
C_{\kappa} & C_{2} & 0 \\
0 & 0 & C_{12}
\end{array}\right)
$$

with

$$
\check{C}=\left(\begin{array}{cc}
C_{1} & C_{\kappa} \\
C_{\kappa} & C_{2}
\end{array}\right), \quad \hat{C}=C_{12}
$$

in the notation introduced right after (8). In the sequel we exclude coupling effects, so that $C_{\kappa}=0$. 


\subsection{Circuit model}

With the notation explained at the beginning of subsection 4.2, a general model for circuits including the devices listed in items 1-6 in subsection 3.2 is defined by the relations

$$
\begin{aligned}
B_{m} v_{m}+B_{w} v_{w}+B_{m c} v_{m c}+B_{m q} v_{m q}+B_{m l} v_{m l}+B_{m \varphi} v_{m \varphi}+B_{j} v_{j} & =-B_{u} v_{u}(t) \\
Q_{m} i_{m}+Q_{w} i_{w}+Q_{m c} i_{m c}+Q_{m q} i_{m q}+Q_{m l} i_{m l}+Q_{m \varphi} i_{m \varphi}+Q_{u} i_{u} & =-Q_{j} i_{j}(t) \\
v_{m} & =\eta_{m}\left(q_{m}, i_{m}\right) \\
i_{w} & =\zeta_{w}\left(\varphi_{w}, v_{w}\right) \\
q_{m c} & =\omega_{m c}\left(\varphi_{m c}, v_{m c}\right) \\
v_{m q} & =\nu_{m q}\left(\sigma_{m q}, q_{m q}\right) \\
\varphi_{m l} & =\theta_{m l}\left(q_{m l}, i_{m l}\right) \\
i_{m \varphi} & =\chi_{m \varphi}\left(\rho_{m \varphi}, \varphi_{m \varphi}\right),
\end{aligned}
$$

together with the differential equations $q_{m}^{\prime}=i_{m}, \varphi_{w}^{\prime}=v_{w}, q_{m c}^{\prime}=i_{m c}, \varphi_{m c}^{\prime}=v_{m c}, q_{m q}^{\prime}=i_{m q}$, $\sigma_{m q}^{\prime}=q_{m q}, \varphi_{m l}^{\prime}=v_{m l}, q_{m l}^{\prime}=i_{m l}, \varphi_{m \varphi}^{\prime}=v_{m \varphi}, \rho_{m \varphi}^{\prime}=\varphi_{m \varphi}$ which govern the dynamics of reactive and mem-devices.

We refer the reader to the Appendix for the definitions and basic properties of the reduced loop and cutset matrices $B$ and $Q$ arising in (17a) and (17b). These equations express Kirchhoff laws; within them, we have split $B$ and $Q$ according to the nature of the different columns, that is, $B_{m}$ comprises the columns of $B$ corresponding to $q$-memristors and current-controlled resistors, $B_{w}$ corresponds to $\varphi$-memristors and voltage-controlled resistors, etc.

Details concerning the circuit model of the running example are given later, but the reader can check e.g. that, orienting all horizontal branches in Figure 1 left-to-right and all vertical ones top-down, a counterclockwise orientation in the loop defined by all three capacitors, the resistors $R_{1}$ and $R_{2}$ and the three voltage sources yields the relation

$$
-v_{r_{1}}-v_{c_{1}}+v_{c_{12}}+v_{c_{2}}+v_{r_{2}}+V_{2}-V_{12}-V_{1}=0 \text {. }
$$

Analogously, as an example of Kirchhoff's current law, the reader can check that the cutset defined by all vertical branches in Figure 1 (cf. also Figure 5) yields

$$
i_{w_{1}}+i_{l_{1}}+i_{r_{1}}+i_{r_{2}}+i_{l_{2}}+i_{w_{2}}=0
$$

\subsection{Fundamental matrices}

The reader can find in the Appendix elementary properties of the reduced loop and cutset matrices $B$ and $Q$. Below we will make use of the so-called fundamental loops and cutsets defined by a spanning tree in a connected circuit: indeed, every cotree branch or link defines a unique (so-called fundamental) loop together with some tree branches or twigs and, analogously, every twig defines a unique (fundamental) cutset together with some links. This confers the corresponding (again called fundamental) loop and cutset matrices the form

$$
B=\left(\begin{array}{ll}
I & K
\end{array}\right), \quad Q=\left(\begin{array}{ll}
-K^{T} & I
\end{array}\right)
$$


for a certain submatrix $K$; find examples in (23), (25). In the choice of the tree arising in the proof of Theorem 1, we will also make use of the fact that, in the absence of loops defined by branches of a certain type (to be referred to as type $A$-branches) and of cutsets of branches of another type (to be called $B$-branches), a spanning tree can be constructed in a way such that all $A$-branches are twigs and all $B$-ones are links [25]. Specifically, we will use this result with voltage sources, memcapacitors and capacitors as $A$-branches, and current sources, meminductors and inductors as $B$-branches.

Now let us split the branches of a connected digraph into four disjoint sets $S_{i}, i=0, \ldots, 3$, where $S_{0}$ and $S_{1}$ are links and $S_{2}$ and $S_{3}$ are twigs of a given spanning tree. Write the fundamental matrices as

$$
B=\left(\begin{array}{cccc}
I_{0} & 0 & K_{00} & K_{01} \\
0 & I_{1} & K_{10} & K_{11}
\end{array}\right), \quad Q=\left(\begin{array}{cccc}
-K_{00}^{T} & -K_{10}^{T} & I_{2} & 0 \\
-K_{01}^{T} & -K_{11}^{T} & 0 & I_{3}
\end{array}\right)
$$

Here, the rows of $B$ (resp. of $Q$ ) correspond to the fundamental loops (resp. cutsets) defined by $S_{0}$ and $S_{1}$ (resp. $S_{2}$ and $S_{3}$ ) branches, in this order; in both matrices, the columns correspond to $S_{0}, S_{1}, S_{2}$ and $S_{3}$ branches.

In Theorem 1, $S_{0}$-branches will correspond to current sources, inductors and meminductors; $S_{1}$-branches will be link resistors and memristors; $S_{2}$-branches will be twig resistors and memristors; and finally $S_{3}$-branches will be voltage sources, capacitors and memcapacitors. For later use, the following lemma captures the effect on the above-introduced matrices of the contraction (short-circuiting) of certain twigs and the removal (open-circuiting) of some links. The running example will be used to illustrate this result later on.

Lemma 1. In the setting described above, the removal of the $S_{0}$-branches and the contraction of the $S_{3}$-branches yield a reduced connected digraph in which $S_{1}$ and $S_{2}$ are the links and twigs of a spanning tree and for which the fundamental matrices read as

$$
\tilde{B}=\left(\begin{array}{ll}
I_{1} & K_{10}
\end{array}\right), \quad \tilde{Q}=\left(\begin{array}{ll}
-K_{10}^{T} & I_{2}
\end{array}\right)
$$

Proof. Note first that after the removal of the $S_{0}$-branches the digraph remains connected, with links defined by $S_{1}$ and twigs by $S_{2}$ and $S_{3}$. This removal does not affect the fundamental links defined by the $S_{1}$ branches, the fundamental matrices of this "intermediate" digraph then being

$$
\hat{B}=\left(\begin{array}{ccc}
I_{1} & K_{10} & K_{11}
\end{array}\right), \quad \hat{Q}=\left(\begin{array}{ccc}
-K_{10}^{T} & I_{2} & 0 \\
-K_{11}^{T} & 0 & I_{3}
\end{array}\right)
$$

Note that the columns defined by $K_{00}^{T}$ and $K_{01}^{T}$ disappear from the cutset matrix because the removal of the $S_{0}$-branches actually modifies the structure of some cutsets.

The dual operation is the contraction of the $S_{3}$-twigs, which yields a reduced digraph with links in $S_{1}$ and twigs in $S_{2}$. This contraction does not affect the fundamental cutsets defined by $S_{2}$-branches and the resulting fundamental matrices are those depicted in (21); again, the fact that $K_{11}$ does not appear in the loop matrix reflects that some of the fundamental loops are modified due to the contraction of the $S_{3}$-branches. 
Fundamental matrices for the running example. In the circuit example of Figure 1, we will work with the spanning tree defined by $C_{1}, C_{12}, C_{2}, R_{1}, V_{1}, V_{12}$ and $V_{2}$ (cf. Figure 4). Note the absence of loops formed by voltage sources, capacitors and/or memcapacitors (say, type$A$ branches) and of cutsets defined by current sources, inductors and/or meminductors (type- $B$ branches; in this case these amount to inductors), which makes it possible to choose the spanning tree in a way such that all voltage sources, capacitors and the memcapacitor are twigs, whereas the inductors are links.

To understand the notion of a fundamental loop/cutset, the reader can e.g. check that the fundamental loop defined by the link $R_{2}$ comprises all twigs, whereas the one defined for instance by the link $L_{2}$ includes the twigs $C_{12}, C_{1}, R_{1}, V_{1}$ and $V_{12}$. Notice the difference e.g. with the loop defined by $L_{2}, C_{2}, R_{2}$ and $V_{2}$, which is not fundamental (with respect to the spanning tree specified in Figure 4) since it includes two links $\left(L_{2}\right.$ and $\left.R_{2}\right)$. Analogously, the fundamental cutset defined by the twig $R_{1}$ includes the links $W_{1}, L_{1}, R_{2}, L_{2}$ and $W_{2}$; cf. Figure $5(\mathrm{a})$ ); by contrast, the cutset defined e.g. by $W_{1}, L_{1}, R_{1}$ and $C_{12}$ is not fundamental because it includes two twigs, namely $R_{1}$ and $C_{12}$. The fundamental cutset defined by the twig $C_{12}$ comprises the links $R_{2}, L_{2}$ and $W_{2}$. For illustrative purposes note e.g. that, in the latter case, the removal of the cutset branches, namely $C_{12}, R_{2}, L_{2}$ and $W_{2}$, yields two connected components, the first one defined by $W_{1}, L_{1}, C_{1}, R_{1}, V_{1}, V_{12}$ and $V_{2}$ and the second one just by $C_{2}$.

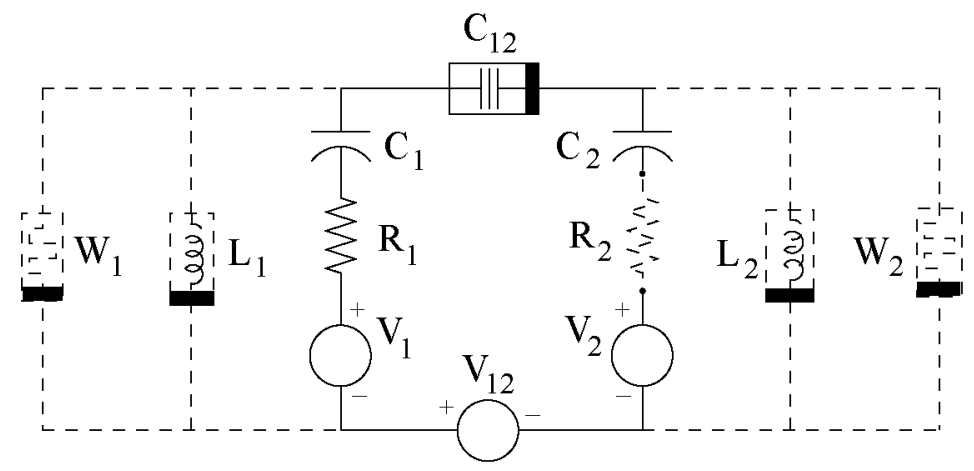

Figure 4: Twigs (continuous line) and links (dashed line) defined by a spanning tree.

Assume that all horizontal branches in Figure 1 are oriented left-to-right and all vertical ones top-down. By enumerating the links in the order $R_{2}, W_{1}, W_{2}, L_{1}, L_{2}$, and the twigs as $R_{1}, C_{1}$, $C_{12}, C_{2}, V_{1}, V_{12}, V_{2}$, the fundamental loop matrix can be checked to read

$$
B=\left(\begin{array}{cccccccccccc}
1 & 0 & 0 & 0 & 0 & -1 & -1 & 1 & 1 & -1 & -1 & 1 \\
0 & 1 & 0 & 0 & 0 & -1 & -1 & 0 & 0 & -1 & 0 & 0 \\
0 & 0 & 1 & 0 & 0 & -1 & -1 & 1 & 0 & -1 & -1 & 0 \\
0 & 0 & 0 & 1 & 0 & -1 & -1 & 0 & 0 & -1 & 0 & 0 \\
0 & 0 & 0 & 0 & 1 & -1 & -1 & 1 & 0 & -1 & -1 & 0
\end{array}\right)
$$

For instance, the first row indicates that the loop defined by the link $R_{2}$ (mind the 1 on top of the first column) includes also $C_{12}, C_{2}$ and $V_{2}$ with the same orientation as the loop itself (the orientation of the loop being defined by that of $R_{2}$ ), and hence the 1 's in the corresponding 
columns, whereas $C_{1}, R_{1}, V_{1}$ and $V_{12}$ enter the loop with the opposite orientation, hence the -1 's in their columns. Note that (23) has the form depicted in (20) for $B$, with

$$
K=\left(\begin{array}{ccccccc}
-1 & -1 & 1 & 1 & -1 & -1 & 1 \\
-1 & -1 & 0 & 0 & -1 & 0 & 0 \\
-1 & -1 & 1 & 0 & -1 & -1 & 0 \\
-1 & -1 & 0 & 0 & -1 & 0 & 0 \\
-1 & -1 & 1 & 0 & -1 & -1 & 0
\end{array}\right)
$$

The corresponding fundamental cutset matrix is

$$
Q=\left(\begin{array}{cccccccccccc}
1 & 1 & 1 & 1 & 1 & 1 & 0 & 0 & 0 & 0 & 0 & 0 \\
1 & 1 & 1 & 1 & 1 & 0 & 1 & 0 & 0 & 0 & 0 & 0 \\
-1 & 0 & -1 & 0 & -1 & 0 & 0 & 1 & 0 & 0 & 0 & 0 \\
-1 & 0 & 0 & 0 & 0 & 0 & 0 & 0 & 1 & 0 & 0 & 0 \\
1 & 1 & 1 & 1 & 1 & 0 & 0 & 0 & 0 & 1 & 0 & 0 \\
1 & 0 & 1 & 0 & 1 & 0 & 0 & 0 & 0 & 0 & 1 & 0 \\
-1 & 0 & 0 & 0 & 0 & 0 & 0 & 0 & 0 & 0 & 0 & 1
\end{array}\right)
$$

With respect to the form depicted in (20), note that the left $7 \times 5$ block of $Q$ is $-K^{T}$, with $K$ given in (24).

In (25), the first row indicates that the fundamental cutset defined by $R_{1}$ includes $R_{2}, W_{1}$, $W_{2}, L_{1}$ and $L_{2}$ (cf. Figure 5(a)), and that all branches are oriented as the cutset itself; this orientation is defined, after the removal of the branches defining the cutset, from the resulting connected component at the top $\left(C_{1}, C_{12}\right.$ and $C_{2}$; cf. Figure 5(b)) to the one at the bottom (defined by $V_{1}, V_{12}$ and $V_{2}$ ). As indicated in Figure 5 , all cutset branches as well as the cutset are oriented top-down, hence the 1's in the cutset matrix.
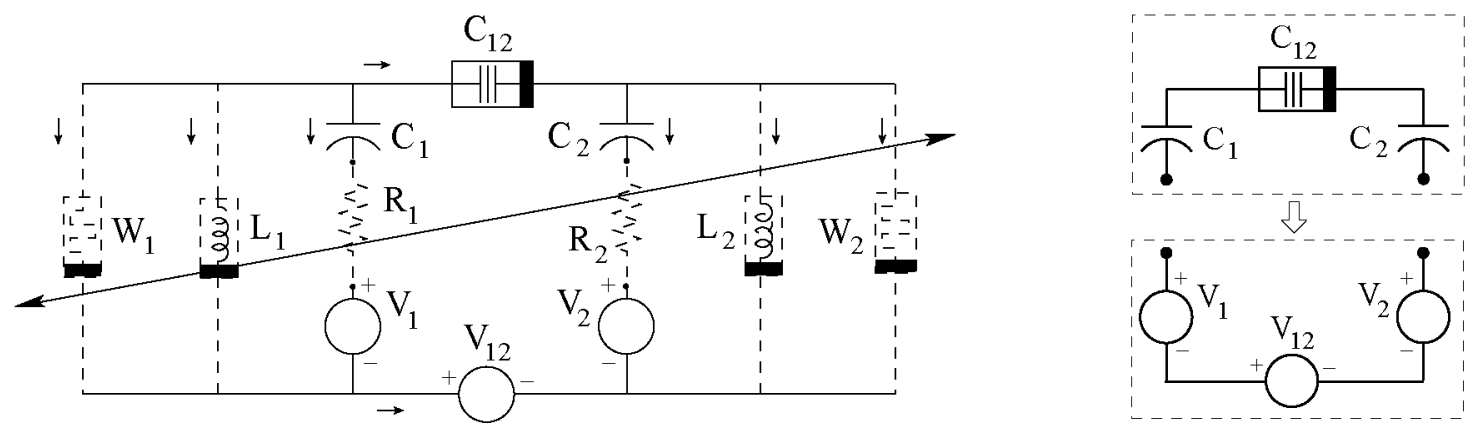

Figure 5: (a) Removing the cutset defined by $W_{1}, L_{1}, R_{1}, R_{2}, L_{2}$ and $W_{2}$ (dashed lines) yields two connected components; (b) orienting the cutset.

This matrices provide an easy writing of Kirchhoff laws as $B v=0, Q i=0$. For instance, the first row of the $B$-matrix yields

$$
v_{r_{2}}-v_{r_{1}}-v_{c_{1}}+v_{c_{12}}+v_{c_{2}}-V_{1}-V_{12}+V_{2}=0,
$$


which is Kirchhoff's voltage law for the fundamental loop defined by $R_{2}$ (compare with (18)). Analogously, the first row of the $Q$-matrix leads to

$$
i_{r_{2}}+i_{w_{1}}+i_{w_{2}}+i_{l_{1}}+i_{l_{2}}+i_{r_{1}}=0
$$

and this is Kirchhoff's current law for the fundamental cutset defined by $R_{1}$ (cf. (19)).

The running example is also useful to illustrate Lemma $1 . S_{0}$-branches are in this case the two inductors $L_{1}$ and $L_{2} ; S_{1}$ are the remaining links defined by the spanning tree of Figure 4 , namely, the resistor $R_{2}$ and the memristors $W_{1}$ and $W_{2}$; there is a unique $S_{2}$-branch which is the twig resistor $R_{1}$; finally, the $S_{3}$-branches are voltage sources, capacitors and the memcapacitor.

After removing the $S_{0}$-branches (the two inductors $L_{1}$ and $L_{2}$ ) we get the circuit displayed in Figure 6. The remaining branches are $R_{2}, W_{1}, W_{2}, R_{1}, C_{1}, C_{12}, C_{2}, V_{1}, V_{12}, V_{2}$. The "intermediate" matrices $\hat{B}, \hat{Q}$ (cf. (22)) are

$$
\hat{B}=\left(\begin{array}{cccccccccc}
1 & 0 & 0 & -1 & -1 & 1 & 1 & -1 & -1 & 1 \\
0 & 1 & 0 & -1 & -1 & 0 & 0 & -1 & 0 & 0 \\
0 & 0 & 1 & -1 & -1 & 1 & 0 & -1 & -1 & 0
\end{array}\right), \hat{Q}=\left(\begin{array}{cccccccccc}
1 & 1 & 1 & 1 & 0 & 0 & 0 & 0 & 0 & 0 \\
1 & 1 & 1 & 0 & 1 & 0 & 0 & 0 & 0 & 0 \\
-1 & 0 & -1 & 0 & 0 & 1 & 0 & 0 & 0 & 0 \\
-1 & 0 & 0 & 0 & 0 & 0 & 1 & 0 & 0 & 0 \\
1 & 1 & 1 & 0 & 0 & 0 & 0 & 1 & 0 & 0 \\
1 & 0 & 1 & 0 & 0 & 0 & 0 & 0 & 1 & 0 \\
-1 & 0 & 0 & 0 & 0 & 0 & 0 & 0 & 0 & 1
\end{array}\right)
$$

where we removed the rows defined by $L_{1}$ and $L_{2}$ from $B$ in (23) and the fourth and fifth

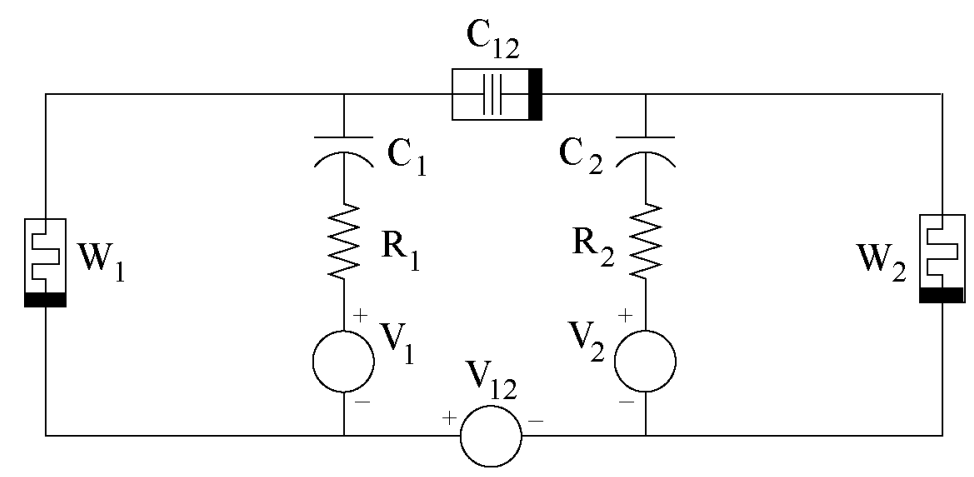

Figure 6: Circuit obtained after removing $L_{1}$ and $L_{2}$ from Figure 1.

columns (the ones corresponding to $L_{1}$ and $L_{2}$ not only from $B$ but also from $Q$ in (25)). Note that the fundamental loops defined by the $S_{1}$-branches $\left(R_{2}, W_{1}, W_{2}\right)$ are not affected by the removal of $L_{1}$ and $L_{2}$; for instance, the first row of $\hat{B}$ reflects the fact that the loop defined by the link $R_{2}$ includes $R_{1}, C_{1}, V_{1}$ and $V_{12}$ with the opposite orientation and $C_{12}, C_{2}$ and $V_{2}$ with the same orientation.

The second step in Lemma 1 is the contraction of $S_{3}$-branches, which in this case means short-circuiting in Figure 6 the memcapacitor, the capacitors and the voltage sources. This leads to the circuit depicted in Figure 7. 


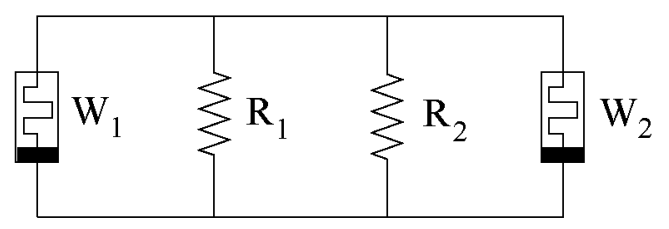

Figure 7: Reduced circuit.

The remaining branches now amount to $R_{2}, W_{1}, W_{2}$, and $R_{1}$, and the matrices $\tilde{B}$ and $\tilde{Q}$ (cf. (21)) read as

$$
\tilde{B}=\left(\begin{array}{cccc}
1 & 0 & 0 & -1 \\
0 & 1 & 0 & -1 \\
0 & 0 & 1 & -1
\end{array}\right), \quad \tilde{Q}=\left(\begin{array}{llll}
1 & 1 & 1 & 1
\end{array}\right)
$$

These are obtained by removing from both $\hat{B}$ and $\hat{Q}$ the last six columns (corresponding to $C_{1}, C_{12}, C_{2}, V_{1}, V_{12}, V_{2}$ ) and, additionally, the last six rows from $\hat{Q}$ which correspond to the fundamental cutsets defined by these six devices.

Note that the matrices in (26) are defined by the links $R_{2}, W_{1}$ and $W_{2}$ and the twig $R_{1}$ in the reduced circuit of Figure 7 . Indeed, $\tilde{B}$ captures the loops defined by the links $R_{2}, W_{1}$ and $W_{2}$ (each one of them defining a loop with the twig $R_{1}$ ), whereas $\tilde{Q}$ just displays the cutset defined by $R_{1}$ (which includes all links $R_{2}, W_{1}$ and $W_{2}$ ).

\subsection{Non-degeneracy}

We are now in a position to state and prove the non-degeneracy theorem.

Theorem 1. Consider a fully nonlinear circuit composed of

- strictly locally passive voltage-and current-controlled resistors and $q$ - and $\varphi$-memristors,

- non-singular voltage- and charge-controlled capacitors and memcapacitors,

- non-singular current- and flux-controlled inductors and meminductors,

besides independent voltage and current sources. In the absence of topological degeneracies, the state dimension of the circuit equals the sum of the state orders of the different devices, and it is therefore defined by the total number of memristors, capacitors and inductors, plus twice the number of memcapacitors and meminductors.

Proof. We assume without loss of generality that the circuit is connected (otherwise, the reasoning applies in the same terms to each connected component). This result relies on the chance to express, using (17), all branch currents and voltages in terms of the dynamic variables, namely $q_{m}, \varphi_{w}, q_{m c}, \varphi_{m c}, q_{m q}, \sigma_{m q}, \varphi_{m l}, q_{m l}, \varphi_{m \varphi}$ and $\rho_{m \varphi}$; indeed, by expressing the branch voltages and currents $v_{m}, i_{m}, v_{w}, i_{w}, v_{m c}, i_{m c}, v_{m q}, i_{m q}, v_{m l}, i_{m l}, v_{m \varphi}$ and $i_{m \varphi}$, plus the current source voltages $v_{j}$ and voltage source currents $i_{u}$, in terms of the aforementioned dynamic variables, 
from the differential equations $q_{m}^{\prime}=i_{m}$ etc. following (17) we would readily get an explicit ODE model for the dynamics.

By definition, $v_{m q}$ and $i_{m \varphi}$ are explicitly given in terms of $\sigma_{m q}, q_{m q}$ and $\rho_{m \varphi}, \varphi_{m \varphi}$ (cf. (17f) and (17h)), whereas $v_{m c}$ and $i_{m l}$ can be expressed, from (17e) and (17g), in terms of $q_{m c}$, $\varphi_{m c}$ and $q_{m l}, \varphi_{m l}$, respectively; this is a consequence of the non-singularity assumptions on $C=\frac{\partial \omega_{m c}}{\partial v_{m c}}, L=\frac{\partial \theta_{m l}}{\partial i_{m l}}$, which make it possible to apply the implicit function theorem in order to solve for $v_{m c}$ and $i_{m l}$ in (17e) and $(17 \mathrm{~g})$. We will denote the corresponding local maps by $v_{m c}=\hat{\omega}_{m c}\left(\varphi_{m c}, q_{m c}\right), i_{m l}=\hat{\theta}_{m l}\left(q_{m l}, \varphi_{m l}\right)$. Additionally, $v_{m}$ and $i_{w}$ are defined in terms of $q_{m}, i_{m}$ and $\varphi_{w}, v_{w}$, respectively (cf. (17c) and (17d)). By grouping together $v_{m l}, v_{m \varphi}$ and $v_{j}$ into a single vector $v_{j l}$ and $i_{m c}, i_{m q}, i_{u}$ into $i_{u c}$, the aforementioned substitutions reduce (17a) and (17b) to

$$
\begin{aligned}
B_{m} \eta_{m}\left(q_{m}, i_{m}\right)+B_{w} v_{w}+B_{m c} \hat{\omega}_{m c}\left(\varphi_{m c}, q_{m c}\right)+B_{m q} \nu_{m q}\left(\sigma_{m q}, q_{m q}\right)+B_{j l} v_{j l}+B_{u} v_{u}(t) & =0 \\
Q_{m} i_{m}+Q_{w} \zeta_{w}\left(\varphi_{w}, v_{w}\right)+Q_{m l} \hat{\theta}_{m l}\left(q_{m l}, \varphi_{m l}\right)+Q_{m \varphi} \chi_{m \varphi}\left(\rho_{m \varphi}, \varphi_{m \varphi}\right)+Q_{u c} i_{u c}+Q_{j} i_{j}(t) & =0 .
\end{aligned}
$$

Here and in (28) below, $B_{j l}$ comprises the columns which correspond to both types of inductors and meminductors, besides current sources, whereas $Q_{u c}$ corresponds to both types of capacitors and memcapacitors, in addition to voltage sources.

Now, the chance to eliminate the remaining variables (that is, $i_{m}, v_{w}, v_{j l}, i_{u c}$ ) is again based on the implicit function theorem, provided that the matrix

$$
A=\left(\begin{array}{cccc}
B_{m} M & B_{w} & B_{j l} & 0 \\
Q_{m} & Q_{w} W & 0 & Q_{u c}
\end{array}\right)
$$

is non-singular; recall that $M=\frac{\partial \eta_{m}}{\partial i_{m}}, W=\frac{\partial \zeta_{w}}{\partial v_{w}}$, so that $A$ is the matrix of partial derivatives of the left-hand sides of (27a)-(27b) with respect to the variables we want to eliminate, that is, $i_{m}, v_{w}, v_{j l}, i_{u c}$.

In order to assess the non-singularity of $A$ in (28), we may assume from the topological nondegeneracy assumption that the reduced loop and cutset matrices $B, Q$ are the fundamental matrices associated with a spanning tree which defines all voltage sources, capacitors and memcapacitors as twigs, and current sources, inductors and meminductors as links; there is no loss of generality in this assumption since any other loop and cutset matrices differ from the given ones only by a non-singular matrix factor (cf. the Appendix) and, therefore, such a change in $B$ and/or $Q$ within (28) would result in the premultiplication by a non-singular matrix factor, which would not affect the eventual non-singular nature of $A$. Now, according to Lemma 1 , by open-circuiting current sources, inductors and meminductors ( $S_{0}$ branches in the notation of Lemma 1), and short-circuiting all voltage sources, capacitors and memcapacitors ( $S_{3}$-branches), we get a reduced circuit described by fundamental loop and cutset matrices

$$
\tilde{B}=\left(\begin{array}{ll}
\tilde{B}_{m} & \tilde{B}_{w}
\end{array}\right), \quad \tilde{Q}=\left(\begin{array}{ll}
\tilde{Q}_{m} & \tilde{Q}_{w}
\end{array}\right),
$$

which are obtained by removing the rows corresponding to the fundamental loops defined by current sources, inductors and meminductors in $B$, those corresponding to fundamental cutsets defined by voltage sources, capacitors and memcapacitors in $Q$, and all the columns corresponding to voltage and current sources and to reactive elements (inductors, meminductors, capacitors 
and memcapacitors) in both matrices. Note that here we do not need to use the link/twig ordering of columns presented earlier in order to introduce fundamental matrices.

By construction, the matrix $A$ in (28) will be non-singular if and only if so it is

$$
\tilde{A}=\left(\begin{array}{cc}
\tilde{B}_{m} M & \tilde{B}_{w} \\
\tilde{Q}_{m} & \tilde{Q}_{w} W
\end{array}\right)
$$

(check the example at the end of this Section). Indeed, each column removed from the $\left(\begin{array}{c}B_{j l} \\ 0\end{array}\right)$ block in (28) just comprises a single non-vanishing entry (equal to 1), since $B$ is assumed to be a fundamental matrix constructed from a tree in a way such that all current sources, inductors and meminductors are links. The rows which are removed from $B$ in this reduction process are precisely those which transform $\left(\begin{array}{lll}B_{m} & B_{w}\end{array}\right)$ into $\left(\begin{array}{ll}\tilde{B}_{m} & \tilde{B}_{w}\end{array}\right)$. Note also that the corresponding remaining rows in $A$ are $\left(\tilde{B}_{m} M \quad \tilde{B}_{w}\right)$. Dual remarks apply to the cutset blocks. Hence, by elementary determinantal properties we have

$$
\operatorname{det} A=\operatorname{det}\left(\begin{array}{cccc}
B_{m} M & B_{w} & B_{j l} & 0 \\
Q_{m} & Q_{w} W & 0 & Q_{u c}
\end{array}\right)= \pm \operatorname{det}\left(\begin{array}{cc}
\tilde{B}_{m} M & \tilde{B}_{w} \\
\tilde{Q}_{m} & \tilde{Q}_{w} W
\end{array}\right)= \pm \operatorname{det} \tilde{A}
$$

Since $M$ and $W$ are positive definite, they are non-singular and their inverses are positive definite as well (cf. the Appendix). We may then compute the product

$$
\begin{aligned}
\left(\begin{array}{cc}
\tilde{B}_{m} M & \tilde{B}_{w} \\
\tilde{Q}_{m} & \tilde{Q}_{w} W
\end{array}\right)\left(\begin{array}{cc}
\tilde{B}_{m}^{T} & M^{-1} \tilde{Q}_{m}^{T} \\
W^{-1} \tilde{B}_{w}^{T} & \tilde{Q}_{w}^{T}
\end{array}\right)= \\
=\left(\begin{array}{cc}
\tilde{B}_{m} M \tilde{B}_{m}^{T}+\tilde{B}_{w} W^{-1} \tilde{B}_{w}^{T} & \tilde{B}_{m} \tilde{Q}_{m}^{T}+\tilde{B}_{w} \tilde{Q}_{w}^{T} \\
\tilde{Q}_{m} \tilde{B}_{m}^{T}+\tilde{Q}_{w} \tilde{B}_{w}^{T} & \tilde{Q}_{m} M^{-1} \tilde{Q}_{m}^{T}+\tilde{Q}_{w} W \tilde{Q}_{w}^{T}
\end{array}\right)
\end{aligned}
$$

Because of the identities $B Q^{T}=0, Q B^{T}=0$ discussed in the Appendix, we have $\tilde{B}_{m} \tilde{Q}_{m}^{T}+$ $\tilde{B}_{w} \tilde{Q}_{w}^{T}=0$ and $\tilde{Q}_{m} \tilde{B}_{m}^{T}+\tilde{Q}_{w} \tilde{B}_{w}^{T}=0$. This confers the matrix product in (31) a block-diagonal structure, with blocks $\tilde{B}_{m} M \tilde{B}_{m}^{T}+\tilde{B}_{w} W^{-1} \tilde{B}_{w}^{T}$ and $\tilde{Q}_{m} M^{-1} \tilde{Q}_{m}^{T}+\tilde{Q}_{w} W \tilde{Q}_{w}^{T}$. Both blocks are positive definite; indeed, provided that $u \neq 0$, we have

$$
u^{T}\left(\tilde{B}_{m} M \tilde{B}_{m}^{T}+\tilde{B}_{w} W^{-1} \tilde{B}_{w}^{T}\right) u=u^{T}\left(\begin{array}{cc}
\tilde{B}_{m} & \tilde{B}_{w}
\end{array}\right)\left(\begin{array}{cc}
M & 0 \\
0 & W^{-1}
\end{array}\right)\left(\begin{array}{c}
\tilde{B}_{m}^{T} \\
\tilde{B}_{w}^{T}
\end{array}\right) u
$$

but since the rows of $\left(\begin{array}{cc}\tilde{B}_{m} & \tilde{B}_{w}\end{array}\right)$ are linearly independent, it follows that

$$
u^{T}\left(\begin{array}{cc}
\tilde{B}_{m} & \tilde{B}_{w}
\end{array}\right)=\left(\left(\begin{array}{c}
\tilde{B}_{m}^{T} \\
\tilde{B}_{w}^{T}
\end{array}\right) u\right)^{T} \neq 0
$$

because the entries of $u^{T}$ define the coefficients of a linear combination of the rows of $\left(\begin{array}{cc}\tilde{B}_{m} & \tilde{B}_{w}\end{array}\right)$. The positive definiteness of blockdiag $\left(M, W^{-1}\right)$ makes the product in (32) positive. The same holds for the block $\tilde{Q}_{m} M^{-1} \tilde{Q}_{m}^{T}+\tilde{Q}_{w} W \tilde{Q}_{w}^{T}$. Being positive definite, both blocks are therefore non-singular. 
Both factors on the left-hand side of (31) must then be non-singular; this conveys the nonsingularity of $\tilde{A}$ and $A$. As indicated above, this makes it possible to apply the implicit function theorem at the operating point to eliminate all branch currents and voltages, yielding a local description of the circuit differential equations in terms of the dynamic variables listed at the beginning of the proof. The number of these variables equals the total number of memristors, capacitors and inductors plus twice the number of memcapacitors and meminductors, and the claim is proved.

Theorem 1 may also be seen, in DAE terms (cf. the Appendix and [22, 27, 28, 29]), as an indexone characterization of second order mem-circuits. We will exploit this point of view later in Section 5 .

Model reduction and state dimension in the running example. The goal of the reduction process is to rewrite all branch voltages and currents in terms of the differential variables $\sigma_{m q}, q_{m q}, \varphi_{w_{1}}, \varphi_{w_{2}}, \varphi_{l_{1}}, \varphi_{l_{2}}, q_{c_{1}}$ and $q_{c_{2}}$. This must be done by using on the one hand the relations (9), (11), (13) and (15) and, on the other, Kirchhoff laws in the form $B v=0$ and $Q i=0$, where $B$ and $Q$ are the loop and cutset matrices given in (23) and (25); here $v$ and $i$ stand for $\left(v_{r_{2}}, v_{w_{1}}, v_{w_{2}}, v_{l_{1}}, v_{l_{2}}, v_{r_{1}}, v_{c_{1}}, v_{m q}, v_{c_{2}}, V_{1}(t), V_{12}(t), V_{2}(t)\right)$ and $\left(i_{r_{2}}, i_{w_{1}}, i_{w_{2}}, i_{l_{1}}, i_{l_{2}}, i_{r_{1}}, i_{c_{1}}, i_{m q}, i_{c_{2}}, i_{u_{1}}, i_{u_{12}}, i_{u_{2}}\right)$, following the order specified right before (23). Once this rewriting is performed, one gets from (10), (12), (14) and (16) an explicit state space model for the dynamics, and hence the state dimension of this model will be eight; two degrees of dynamic freedom are contributed by the memcapacitor and one by each one of the memristors, capacitors and inductors. This can be proved feasible as a consequence of the nondegeneracy result stated in Theorem 1; in the sequel we check this in practice.

To do so, notice that the reduction (27) in this case takes the form

$$
\begin{array}{r}
B_{m} M i_{m}+B_{w} v_{w}+B_{l} v_{l}+B_{c} C^{-1} q_{c}+B_{m q} \nu_{m q}\left(\sigma_{m q}, q_{m q}\right)+B_{u} v_{u}(t)=0 \\
Q_{m} i_{m}+Q_{w} W\left(\varphi_{w}\right) v_{w}+Q_{l} \chi\left(\varphi_{l}\right)+Q_{c} i_{c}+Q_{m q} i_{m q}+Q_{u} i_{u}=0
\end{array}
$$

where

$$
\begin{gathered}
B_{m}=\left(\begin{array}{ll}
-1 & 1 \\
-1 & 0 \\
-1 & 0 \\
-1 & 0 \\
-1 & 0
\end{array}\right), B_{w}=\left(\begin{array}{ll}
0 & 0 \\
1 & 0 \\
0 & 1 \\
0 & 0 \\
0 & 0
\end{array}\right), B_{l}=\left(\begin{array}{ll}
0 & 0 \\
0 & 0 \\
0 & 0 \\
1 & 0 \\
0 & 1
\end{array}\right), B_{c}=\left(\begin{array}{ll}
-1 & 1 \\
-1 & 0 \\
-1 & 0 \\
-1 & 0 \\
-1 & 0
\end{array}\right), B_{m q}=\left(\begin{array}{l}
1 \\
0 \\
1 \\
0 \\
1
\end{array}\right), \\
B_{u}=\left(\begin{array}{rrr}
-1 & -1 & 1 \\
-1 & 0 & 0 \\
-1 & -1 & 0 \\
-1 & 0 & 0 \\
-1 & -1 & 0
\end{array}\right), Q_{m}=\left(\begin{array}{rr}
1 & 1 \\
0 & 1 \\
0 & -1 \\
0 & -1 \\
0 & 1 \\
0 & 1 \\
0 & -1
\end{array}\right), Q_{w}=\left(\begin{array}{rr}
1 & 1 \\
1 & 1 \\
0 & -1 \\
0 & 0 \\
1 & 1 \\
0 & 1 \\
0 & 0
\end{array}\right), Q_{l}=\left(\begin{array}{rr}
1 & 1 \\
1 & 1 \\
0 & -1 \\
0 & 0 \\
1 & 1 \\
0 & 1 \\
0 & 0
\end{array}\right)
\end{gathered}
$$


and $Q_{c}, Q_{m q}$ and $Q_{u}$ are given accordingly from the identity block defined by the last seven columns of (25). Note that all these submatrices are constructed from the columns of $B$ and $Q$ in (23)-(25) defined by $R_{1}, R_{2} ; W_{1}, W_{2} ; L_{1}, L_{2} ; C_{1}, C_{2} ; C_{12}$; and $V_{1}, V_{12}, V_{2}$, respectively. Additionally, $M=\operatorname{diag}\left(R_{1}, R_{2}\right), W\left(\varphi_{w}\right)=\operatorname{diag}\left(W_{1}\left(\varphi_{w_{1}}\right), W_{2}\left(\varphi_{w_{2}}\right)\right)$ where $\varphi_{w}=\left(\varphi_{w_{1}} \varphi_{w_{2}}\right)^{T}$, $\chi\left(\varphi_{l}\right)=\left(\chi_{1}\left(\varphi_{l_{1}}\right) \chi_{2}\left(\varphi_{l_{2}}\right)\right)^{T}$ with $\varphi_{l}=\left(\varphi_{l_{1}} \varphi_{l_{2}}\right)^{T}$, and $C=\operatorname{diag}\left(C_{1}, C_{2}\right)$.

The goal is to express

$$
i_{m}=\left(\begin{array}{c}
i_{r_{1}} \\
i_{r_{2}}
\end{array}\right), v_{w}=\left(\begin{array}{c}
v_{w_{1}} \\
v_{w_{2}}
\end{array}\right), v_{l}=\left(\begin{array}{c}
v_{l_{1}} \\
v_{l_{2}}
\end{array}\right), i_{c}=\left(\begin{array}{c}
i_{c_{1}} \\
i_{c_{2}}
\end{array}\right), i_{m q}, i_{u}=\left(\begin{array}{c}
i_{u_{1}} \\
i_{u_{12}} \\
i_{u_{2}}
\end{array}\right)
$$

in terms of

$$
q_{c}=\left(\begin{array}{c}
q_{c_{1}} \\
q_{c_{2}}
\end{array}\right), v_{u}(t)=\left(\begin{array}{c}
V_{1}(t) \\
V_{12}(t) \\
V_{2}(t)
\end{array}\right)
$$

and $\varphi_{w}, \varphi_{l}, \sigma_{m q}, q_{m q}$. To achieve this, we need to check that the matrix of coefficients of $i_{m}, v_{w}, v_{l}, i_{c}, i_{m q}, i_{u}$ defined by (33) is non-singular (notice that in this case the system is linear in the variables $\left.i_{m}, v_{w}, v_{l}, i_{c}, i_{m q}, i_{u}\right)$. This matrix of coefficients is the $A$-matrix depicted in (28), which in this case takes the form

$$
A=\left(\begin{array}{cccccc}
B_{m} M & B_{w} & B_{l} & 0 & 0 & 0 \\
Q_{m} & Q_{w} W & 0 & Q_{c} & Q_{m q} & Q_{u}
\end{array}\right)
$$

and reads as

$$
A=\left(\begin{array}{cccccccccccc}
-R_{1} & R_{2} & 0 & 0 & 0 & 0 & 0 & 0 & 0 & 0 & 0 & 0 \\
-R_{1} & 0 & 1 & 0 & 0 & 0 & 0 & 0 & 0 & 0 & 0 & 0 \\
-R_{1} & 0 & 0 & 1 & 0 & 0 & 0 & 0 & 0 & 0 & 0 & 0 \\
-R_{1} & 0 & 0 & 0 & 1 & 0 & 0 & 0 & 0 & 0 & 0 & 0 \\
-R_{1} & 0 & 0 & 0 & 0 & 1 & 0 & 0 & 0 & 0 & 0 & 0 \\
1 & 1 & W_{1} & W_{2} & 0 & 0 & 0 & 0 & 0 & 0 & 0 & 0 \\
0 & 1 & W_{1} & W_{2} & 0 & 0 & 1 & 0 & 0 & 0 & 0 & 0 \\
0 & -1 & 0 & -W_{2} & 0 & 0 & 0 & 0 & 1 & 0 & 0 & 0 \\
0 & -1 & 0 & 0 & 0 & 0 & 0 & 1 & 0 & 0 & 0 & 0 \\
0 & 1 & W_{1} & W_{2} & 0 & 0 & 0 & 0 & 0 & 1 & 0 & 0 \\
0 & 1 & 0 & W_{2} & 0 & 0 & 0 & 0 & 0 & 0 & 1 & 0 \\
0 & -1 & 0 & 0 & 0 & 0 & 0 & 0 & 0 & 0 & 0 & 1
\end{array}\right)
$$

where the bottom-right $7 \times 6$ submatrix is defined by $Q_{c}, Q_{m q}, Q_{u}$, that is, by the entries of $Q$ defined by $C_{1}, C_{2}, C_{12}, V_{1}, V_{12}, V_{2}$ (in this order). Expanding the determinant successively along the last eight columns (note that there is a single " 1 " in each one of them), this matrix is checked to be non-singular if and only if so it is

$$
\tilde{A}=\left(\begin{array}{cccc}
-R_{1} & R_{2} & 0 & 0 \\
-R_{1} & 0 & 1 & 0 \\
-R_{1} & 0 & 0 & 1 \\
1 & 1 & W_{1} & W_{2}
\end{array}\right)
$$


which is obtained after removing the last eight columns of $A$ and the rows defined by these 1-entries. The determinant of $\tilde{A}$ is

$$
-\left(R_{1}+R_{2}+R_{1} R_{2}\left(W_{1}+W_{2}\right)\right)
$$

and the passivity assumption on resistive and memristive devices, which implies $R_{k}>0, W_{k}>0$ $(k=1,2)$, makes this determinant non-null. This guarantees the non-singularity of $\tilde{A}$, hence of $A$, and the feasibility of the whole reduction process.

Note finally that $\tilde{A}$ has the structure depicted in (29), with

$$
\tilde{B}_{m}=\left(\begin{array}{ll}
-1 & 1 \\
-1 & 0 \\
-1 & 0
\end{array}\right), \quad \tilde{B}_{w}=\left(\begin{array}{ll}
0 & 0 \\
1 & 0 \\
0 & 1
\end{array}\right), \tilde{Q}_{m}=\left(\begin{array}{ll}
1 & 1
\end{array}\right), \tilde{Q}_{w}=\left(\begin{array}{ll}
1 & 1
\end{array}\right)
$$

Here $\tilde{B}_{m}$ and $\tilde{Q}_{m}$ come from the last and first columns of $\tilde{B}$ and $\tilde{Q}$ in (26), whereas $\tilde{B}_{w}$ and $\tilde{Q}_{w}$ come from the second and third ones, following the branch ordering $R_{2}, W_{1}, W_{2}, R_{1}$ specified there.

\section{Dynamical features of a family of R-MC-ML circuits}

In this Section we examine a family of nonlinear circuits exhibiting rich dynamics and defined by the series connection of a charge-controlled memcapacitor, a flux-controlled meminductor and a linear resistor closed on a short circuit. The failure of some of the assumptions supporting Theorem 1 will lead to structural changes and interesting bifurcation phenomena. Further analysis of this type of behavior is in the scope of future research.

We assume as in [5] that the mem-devices are defined by two-variable differentiable relations, namely $\varphi_{m c}=\alpha\left(\sigma_{m c}\right)$ (together with $\sigma_{m c}^{\prime}=q_{m c}, q_{m c}^{\prime}=i_{m c}, \varphi_{m c}^{\prime}=v_{m c}$ ) for the memcapacitor, and $q_{m l}=\beta\left(\rho_{m l}\right)$ for the meminductor (besides $\rho_{m l}^{\prime}=\varphi_{m l}, \varphi_{m l}^{\prime}=v_{m l}, q_{m l}^{\prime}=i_{m l}$ ). Both devices are assumed to be strictly locally passive (so that $\alpha^{\prime}\left(\sigma_{m c}\right)>0$ and $\beta^{\prime}\left(\rho_{m l}\right)>0$ everywhere) and $\alpha$ and $\beta$ are assumed to be surjective (a function $f: \mathbb{R} \rightarrow \mathbb{R}$ is said to be surjective or onto if for all real number $y$ there exists an $x$ such that $f(x)=y$, a condition equivalent under the assumptions above on $\alpha$ and $\beta$ to $\left.\lim _{x \rightarrow-\infty} f(x)=-\infty, \lim _{x \rightarrow \infty} f(x)=\infty\right)$. We will first suppose that the resistor in series is a linear one with resistance $R$, and later on with conductance $G$, to examine what happens if $R$ or $G$ eventually vanish and become negative.

Focus first on the case defined by a resistor with resistance $R$. The state order of both mem-devices is two and, at least when $R>0$, the state dimension of the circuit should be four, according to Theorem 1; actually, a state equation will be formulated in terms of $\sigma_{m c}, q_{m c}$, $\rho_{m l}$ and $\varphi_{m l}$. To see this in practice, the reader should not simply disregard $\varphi_{m c}$ and $q_{m l}$ as output variables (defined by the relations $\varphi_{m c}=\alpha\left(\sigma_{m c}\right)$ and $q_{m l}=\beta\left(\rho_{m l}\right)$ ), since these relations pose hidden constraints among the remaining circuit variables. Both $\varphi_{m c}$ and $q_{m l}$ can be indeed eliminated, but not before differentiating the characteristic relations of both mem-devices to obtain $v_{m c}=\alpha^{\prime}\left(\sigma_{m c}\right) q_{m c}, i_{m l}=\beta^{\prime}\left(\rho_{m l}\right) \varphi_{m l}$, which make it possible to formulate the state-space 
model

$$
\begin{aligned}
\sigma_{m c}^{\prime} & =q_{m c} \\
q_{m c}^{\prime} & =\beta^{\prime}\left(\rho_{m l}\right) \varphi_{m l} \\
\rho_{m l}^{\prime} & =\varphi_{m l} \\
\varphi_{m l}^{\prime} & =-\alpha^{\prime}\left(\sigma_{m c}\right) q_{m c}-R \beta^{\prime}\left(\rho_{m l}\right) \varphi_{m l} .
\end{aligned}
$$

This system has indeed state dimension four. In this case, $R$ becoming null or negative does not affect the state dimension, although from the dynamical point of view the vanishing of $R$ will result in the bifurcation phenomenon addressed below.

System (37) has two peculiar features. First, equilibria are not isolated, defining instead an equilibrium surface given by $q_{m c}=\varphi_{m l}=0$ (lines of equilibria have been already observed in circuits with memristors $[10,19,26])$. Second, the dynamics is foliated by a family of invariant surfaces which is parameterized by two constants $k_{1}, k_{2}$. To see this, recast $(37 \mathrm{~b})$ as $q_{m c}^{\prime}=$ $\beta^{\prime}\left(\rho_{m l}\right) \varphi_{m l}=\left(\beta\left(\rho_{m l}\right)\right)^{\prime}$, that is, $\left(q_{m c}-\beta\left(\rho_{m l}\right)\right)^{\prime}=0$. This means that $q_{m c}-\beta\left(\rho_{m l}\right)$ is a quantity $k_{1}$ which is conserved along trajectories. Analogously, from $(37 \mathrm{~d})$ it follows that $\varphi_{m l}+\alpha\left(\sigma_{m c}\right)+$ $R \beta\left(\rho_{m l}\right)$ is also an invariant quantity $k_{2}$. Note that the constants $k_{1}$ and $k_{2}$ are defined from the initial values of a given trajectory as $k_{1}=q_{m c}(0)-\beta\left(\rho_{m l}(0)\right)$ and $k_{2}=\varphi_{m l}(0)+\alpha\left(\sigma_{m c}(0)\right)+$ $R \beta\left(\rho_{m l}(0)\right)$.

The conditions $q_{m c}-\beta\left(\rho_{m l}\right)=k_{1}$ and $\varphi_{m l}+\alpha\left(\sigma_{m c}\right)+R \beta\left(\rho_{m l}\right)=k_{2}$ together specify a twoparameter family of surfaces which are invariant for the dynamics. Moreover, all these invariant surfaces intersect the equilibrium surface in a single point $\left(\sigma_{m c}, q_{m c}, \rho_{m l}, \varphi_{m l}\right)=\left(\sigma_{m c}^{*}, 0, \rho_{m l}^{*}, 0\right)$, with $\sigma_{m c}^{*}$ and $\rho_{m l}^{*}$ being determined by the relations $\beta\left(\rho_{m l}^{*}\right)=-k_{1}$ and $\alpha\left(\sigma_{m c}^{*}\right)=R k_{1}+k_{2}$; note that the working assumptions on $\alpha, \beta$ yield a unique solution for these equations for each fixed value of $k_{1}, k_{2}$.

The two-dimensional dynamics in such invariant surfaces can be described in terms of $\sigma_{m c}$ and $\rho_{m l}$ from (37a) and (37c), namely

$$
\begin{aligned}
\sigma_{m c}^{\prime} & =\beta\left(\rho_{m l}\right)+k_{1} \\
\rho_{m l}^{\prime} & =-\alpha\left(\sigma_{m c}\right)-R \beta\left(\rho_{m l}\right)+k_{2} .
\end{aligned}
$$

The whole foliation of the phase portrait is not affected by the eventual vanishing of $R$; however, when $R$ becomes null and eventually negative, the stability of the equilibrium in all invariant surfaces changes. This is an easy consequence of the expression for the eigenvalues of the matrix of partial derivatives of the right-hand side of (37), namely

$$
\left(\begin{array}{cccc}
0 & 1 & 0 & 0 \\
0 & 0 & 0 & \beta^{\prime} \\
0 & 0 & 0 & 1 \\
0 & -\alpha^{\prime} & 0 & -R \beta^{\prime}
\end{array}\right)
$$

at equilibrium (where $q_{m c}=\varphi_{m l}=0$ ). For notational simplicity, here and in the sequel we write $\alpha^{\prime}, \beta^{\prime}$ as an abbreviation for $\alpha^{\prime}\left(\sigma_{m c}^{*}\right)$ and $\beta^{\prime}\left(\rho_{m l}^{*}\right)$, respectively. The characteristic polynomial of $(39)$ is

$$
\lambda^{2}\left(\lambda^{2}+R \beta^{\prime} \lambda+\alpha^{\prime} \beta^{\prime}\right)
$$


which has two zero eigenvalues (as expected from the existence of an equilibrium surface) and two non-vanishing ones, namely,

$$
\frac{1}{2}\left(-R \beta^{\prime} \pm \sqrt{\left(R \beta^{\prime}\right)^{2}-4 \alpha^{\prime} \beta^{\prime}}\right)
$$

These two eigenvalues characterize the linear stability properties of the (unique) equilibrium in each invariant surface, and can be equivalently obtained from the matrix of partial derivatives of the right-hand side of (38).

It is easy to check that if $R$ is small, specifically if $|R|<2 \sqrt{\alpha^{\prime} / \beta^{\prime}}$ then the radicand in (40) is negative. This means that for small $R$ the eigenvalues have a non-trivial imaginary part $\pm i \sqrt{\left|\left(R \beta^{\prime}\right)^{2} / 4-\alpha^{\prime} \beta^{\prime}\right|}$. The real part is $-R \beta^{\prime} / 2$ and therefore as $R$ decreases through zero the pair of eigenvalues crosses the imaginary axis towards the right-half plane, yielding a stability loss as in the well-known Hopf bifurcation.

The case in which the resistor is defined by the conductance $G$ leads to a more intricate local behavior near $G=0$ (if $G \neq 0$ then the dynamics amounts to the case discussed above, with $R=1 / G$ ). Now the dynamics near $G=0$ is described by the DAE (cf. the Appendix)

$$
\begin{aligned}
\sigma_{m c}^{\prime} & =q_{m c} \\
q_{m c}^{\prime} & =\beta^{\prime}\left(\rho_{m l}\right) \varphi_{m l} \\
\rho_{m l}^{\prime} & =\varphi_{m l} \\
\varphi_{m l}^{\prime} & =-\alpha^{\prime}\left(\sigma_{m c}\right) q_{m c}-v_{g} \\
0 & =\beta^{\prime}\left(\rho_{m l}\right) \varphi_{m l}-G v_{g}
\end{aligned}
$$

Note that there is not only an additional equation (the restriction (41e), where solutions lie) but also a variable which is not present in (37), namely, the voltage drop at the resistor $v_{g}$. Notice that we cannot recast (41) as an explicit ODE such as (37) holding for all values of $G$ on any neighborhood of 0 , because such description is not feasible for $G=0$. Therefore, the analysis of the transition through $G=0$ must be performed in terms of (41). For $G \neq 0$, invariant surfaces are given by the relations $q_{m c}-\beta\left(\rho_{m l}\right)=k_{1}$ and $G \varphi_{m l}+G \alpha\left(\sigma_{m c}\right)+\beta\left(\rho_{m l}\right)=k_{2}$, and equilibria are unique in each of these invariant surfaces; they are defined by the values $q_{m c}=\varphi_{m l}=v_{g}=0$, together with $\sigma_{m c}^{*}$ and $\rho_{m l}^{*}$ which are uniquely determined by the relations $\beta\left(\rho_{m l}^{*}\right)=-k_{1}$ and $G \alpha\left(\sigma_{m c}^{*}\right)=k_{1}+k_{2}$.

The case $G \leq 0$ rules out the passivity assumption on resistors in Theorem 1 . Without this assumption, we are no longer guaranteed that a state-space description in terms of an explicit ODE is feasible; indeed, when $G$ vanishes there will be a drop in the number of finite eigenvalues characterizing the linearized problem and this will be responsible for another type of bifurcation, as detailed below. It is worth emphasizing that the case $G=0$ models an open-circuit in the linearized circuit.

The eigenvalues of the linearized problem are given by the spectrum of the matrix pencil (cf. 
$[22,27,28,29,30]$ and the Appendix) associated with (41), namely

$$
\lambda\left(\begin{array}{ccccc}
1 & 0 & 0 & 0 & 0 \\
0 & 1 & 0 & 0 & 0 \\
0 & 0 & 1 & 0 & 0 \\
0 & 0 & 0 & 1 & 0 \\
0 & 0 & 0 & 0 & 0
\end{array}\right)-\left(\begin{array}{ccccc}
0 & 1 & 0 & 0 & 0 \\
0 & 0 & 0 & \beta^{\prime} & 0 \\
0 & 0 & 0 & 1 & 0 \\
0 & -\alpha^{\prime} & 0 & 0 & -1 \\
0 & 0 & 0 & \beta^{\prime} & -G
\end{array}\right),
$$

the determinant of which is

$$
\lambda^{2}\left(G \lambda^{2}+\beta^{\prime} \lambda+\alpha^{\prime} \beta^{\prime} G\right)
$$

Again, $\alpha^{\prime}, \beta^{\prime}$ stand for $\alpha^{\prime}\left(\sigma_{m c}^{*}\right)$ and $\beta^{\prime}\left(\rho_{m l}^{*}\right)$, respectively.

For any value of $G$ two eigenvalues do vanish, again consistently with the presence of an equilibrium surface. If $G \neq 0$, there are two additional finite eigenvalues given by

$$
\frac{-\beta^{\prime} \pm \sqrt{\left(\beta^{\prime}\right)^{2}-4 \alpha^{\prime} \beta^{\prime} G^{2}}}{2 G} .
$$

Provided that $G$ is small (specifically, if $|G|<\sqrt{\beta^{\prime}} /\left(2 \sqrt{\alpha^{\prime}}\right)$ ), the radicand in (43) is positive and hence for small, non-vanishing $G$ both eigenvalues are real. On the other hand, if $G=0$ then the determinant of (42) amounts to $\lambda^{3} \beta^{\prime}$; now there is only one additional finite eigenvalue, which also vanishes. This corresponds to a double eigenvalue transition in (43) as $G$ decreases through zero; one of the pencil eigenvalues diverges through $\infty$, whereas the other one crosses the origin along the real axis. In more detail, as $G>0$ (resp. $G<0$ ) tends to zero, one real eigenvalue tends to $-\infty$ (resp. to $+\infty$ ). At the bifurcation value $G=0$ the matrix pencil displays one additional infinite eigenvalue (cf. the Appendix): this divergence through $\infty$ is reminiscent of a singularity-induced bifurcation $[31,32]$, which in this case coexists with a (say, regular) zero-eigenvalue transition.

This combined regular/singular bifurcation has not been reported in the literature and requires further study. A detailed analysis of this behavior and of other qualitative phenomena arising in the dynamics of second order mem-circuits is in the scope of future research.

\section{Concluding remarks}

Memory devices are likely to play a relevant role in the evolution of electronics. The second-order circuit elements here discussed complete the extension of classical nonlinear devices, and their classification in terms of their differential and state orders should be of help in future theoretical developments. A systematic analysis of the dynamics of mem-circuits including second order devices, addressing stability issues and bifurcations, as well as the numerical treatment of such circuits, define promising lines of future research.

\section{Appendix}

We compile below some auxiliary mathematical notions and results which have been used throughout the document. 
Digraphs; loop and cutset matrices. Given an electrical circuit, an underlying directed graph (or digraph) is defined after choosing an orientation in every branch, which specifies the flow direction for positive currents. In a digraph, a path connecting two nodes $v_{0}$ and $v_{l}$ is a sequence $\left(v_{0}, e_{1}, v_{1}, \ldots, v_{l-1}, e_{l}, v_{l}\right)$ in which the branch $e_{i}$ is incident with the nodes $v_{i-1}$ and $v_{i}$ for $1 \leq i \leq l$; note that $e_{i}$ can be directed from $v_{i-1}$ to $v_{i}$ or from $v_{i}$ to $v_{i-1}$. A loop or cycle is a closed path without self-intersections. A loop with $l \geq 2$ can be given two different orientations by specifying one of two orders in the aforementioned sequence, say $\left(v_{0}, e_{1}, v_{1}, \ldots, v_{l-1}, e_{l}, v_{l}\right)$ or $\left(v_{l}, e_{l}, v_{l-1}, \ldots, v_{1}, e_{1}, v_{0}\right)$; note that $v_{0}=v_{l}$. A digraph is said to be connected if for every pair of vertices there exists a path connecting them. A connected component is a maximal connected subgraph of a given digraph, that is, a connected subgraph which is not strictly contained in another connected subgraph. In a connected digraph, a spanning tree is a connected subgraph which includes all nodes and has no loops; a spanning tree divides the set of branches in two: twigs (or tree branches) and links (or cotree branches). A tree is usually identified with the set of twigs, disregarding the nodes. An electrical circuit is said to be connected if its underlying digraph is connected.

Given a digraph with $n$ nodes, $b$ branches and $k$ connected components, the loop matrix is defined as $\bar{B}=\left(b_{i j}\right) \in \mathbb{R}^{l \times b}$ ( $l$ standing for the total number of loops) with

$$
b_{i j}=\left\{\begin{aligned}
1 & \text { if branch } j \text { is in loop } i \text { with the same orientation } \\
-1 & \text { if branch } j \text { is in loop } i \text { with the opposite orientation } \\
0 & \text { if branch } j \text { is not in loop } i
\end{aligned}\right.
$$

The matrix $\bar{B}$ can be proved to have rank $b-n+k$ (see e.g. $[33,34]$ ), and a reduced loop matrix $B$ is any submatrix defined by $b-n+k$ independent rows of $\bar{B}$; in a connected digraph $(k=1), b-n+1$ is the number of links defined by any spanning tree, and in this case the dimensions of $B$ are therefore the number of links times the total number of branches. The rows of $\bar{B}$ span the so-called cycle space (cf. [33]) and the choice of $b-n+k$ independent rows corresponds to the choice of a basis of this space. It follows that if $B_{1}$ and $B_{2}$ are two reduced loop matrices (corresponding to the choice of two different bases), then it holds that $B_{1}=M B_{2}$ for a non-singular matrix $M$. The same remarks will apply to the matrix $Q$ introduced below.

Analogously, the entries of the cutset matrix $\bar{Q}=\left(q_{i j}\right) \in \mathbb{R}^{c \times b}$ (where $c$ stands for the total number of cutsets) are given by

$$
q_{i j}=\left\{\begin{aligned}
1 & \text { if branch } j \text { is in cutset } i \text { with the same orientation } \\
-1 & \text { if branch } j \text { is in cutset } i \text { with the opposite orientation } \\
0 & \text { if branch } j \text { is not in cutset } i
\end{aligned}\right.
$$

Recall that a subset $S$ of the set of branches of a digraph is a cutset if the removal of $S$ increases the number of connected components of the digraph, and it is minimal with respect to this property, that is, the removal of any proper subset of $S$ does not increase this number. Specifically, the removal of a cutset splits a connected component in two, and all cutset branches connect these two split components; a cutset is oriented just by directing it from one of these split connected components towards the other; cf. Figure 5(b), where the cutset is oriented from 
the connected component at the top towards the one at the bottom. The cutset matrix has rank $n-k$ and a reduced cutset matrix $Q$ is any submatrix defined by $n-k$ independent rows of $\bar{Q}$. In a connected digraph $(k=1), n-1$ is the number of twigs in any spanning tree, and in this case the dimensions of $Q$ are the number of twigs times the total number of branches.

The reduced loop and cutset matrices make it possible to express Kirchhoff laws in the form $B v=0, Q i=0$. On the other hand, by construction of $B$ and $Q$ and as detailed e.g. in [35] (Section 7.4), the identities $B Q^{T}=Q B^{T}=0$ hold for any digraph (the reader can check these identities in practice using e.g. the matrices $B$ and $Q$ from (23)-(25) or $\tilde{B}$ and $\tilde{Q}$ from (26)). These relations express the orthogonality of the so-called cut space $\operatorname{im} Q^{T}$ and the cycle space $\operatorname{im} B^{T}$, where im denotes the image space (i.e. the range); cf. [33]. From these identities it follows immediately that $\operatorname{im} Q^{T} \subseteq \operatorname{ker} B$ (where ker denotes the kernel) and $\operatorname{im} B^{T} \subseteq \operatorname{ker} Q$. Additionally, because of the property $\operatorname{rk} B=b-n+k$ (where rk stands for "rank") we have $\operatorname{dim}$ ker $B=n-k$ (here $\operatorname{dim}$ means "dimension"); since $\operatorname{rk} Q=\operatorname{rk} Q^{T}=n-k$, it follows that both spaces $\operatorname{im} Q^{T}$ and $\operatorname{ker} B$ have the same dimension and therefore $\operatorname{im} Q^{T} \subseteq \operatorname{ker} B$ actually implies $\operatorname{im} Q^{T}=\operatorname{ker} B$. We may check in the same manner that the identity $\operatorname{im} B^{T}=\operatorname{ker} Q$ also holds.

The relation $\operatorname{ker} B=\operatorname{im} Q^{T}$ implies that, if $B v=0$, then $v=Q^{T} u$ for a certain vector $u$. Analogously, if $Q i=0$ holds, then $i=B^{T} j$ for some vector $j$. These identities yield $i^{T} v=j^{T} B Q^{T} u=0$, that is $i^{T} v=0$. Note that this is Tellegen's theorem, which says that $i^{T} v=0$ for any pair of vectors $i$ and $v$ satisfying Kirchhoff laws.

The Implicit Function Theorem. Let $f: \mathbb{R}^{n+m} \rightarrow \mathbb{R}^{m}$ be a $C^{1}$ map. Assume that $f\left(x_{0}, y_{0}\right)=$ 0 and that the matrix of partial derivatives $\frac{\partial f}{\partial y}\left(x_{0}, y_{0}\right)$ of $f$ with respect to the $y$-variables is nonsingular. Then there exist certain neighborhoods $U \subseteq \mathbb{R}^{n+m}, V \subseteq \mathbb{R}^{n}, W \subseteq \mathbb{R}^{m}$ of $\left(x_{0}, y_{0}\right)$, $x_{0}$ and $y_{0}$, respectively, and a locally defined $C^{1}$ map $g: V \rightarrow W$ such that $f(x, y)=0$ on $U$ if and only if $y=g(x)$ for $x \in V$. For instance, if $f: \mathbb{R}^{2} \rightarrow \mathbb{R}$ reads $x^{2}+y^{2}-c$ for some positive constant $c$, and we fix a vector $\left(x_{0}, y_{0}\right)$ such that $x_{0}^{2}+y_{0}^{2}=c$ with $y_{0} \neq 0$, we have $\frac{\partial f}{\partial y}\left(x_{0}, y_{0}\right)=2 y_{0} \neq 0$; this means that provided $y_{0} \neq 0$ we may write locally $y$ in terms of $x$ to solve $x^{2}+y^{2}=c$; namely, one gets $y=+\sqrt{c-x^{2}}$ if $y_{0}>0$ or $y=-\sqrt{c-x^{2}}$ if $y_{0}<0$, with $x \in(-\sqrt{c},+\sqrt{c})$ in both cases. By contrast, solving $x^{2}+y^{2}=c$ for $y$ near $y_{0}=0$ in terms of a $C^{1}$ function of $x$ is not possible either on a neighborhood of $x_{0}=-\sqrt{c}$ or of $x_{0}=+\sqrt{c}$.

Positive definite matrices and passivity. A matrix $P \in \mathbb{R}^{n \times n}$ is positive definite if $u^{T} P u>0$ for any non-vanishing vector $u \in \mathbb{R}^{n}$. A positive definite matrix $P$ is non-singular, because if $P u=0$ then $u^{T} P u=0$ and therefore $u=0$. The inverse of a positive definite matrix is also positive definite; indeed, by writing $P^{-1} u=v$ we have $u=P v$ and then

$$
u^{T} P^{-1} u=v^{T} P^{T} P^{-1} u=v^{T} P^{T} v=v^{T} P v>0
$$

because $v \neq 0$ if $u \neq 0$. Note that although $P$ need not be symmetric, the identity $v^{T} P^{T} v=v^{T} P v$ holds since $v^{T} P v$ is a scalar and therefore $v^{T} P v=\left(v^{T} P v\right)^{T}=v^{T} P^{T} v$.

Positive definite matrices describe strictly passive devices. Given e.g. a set of resistors defined by a resistance matrix $R$, the dot product $i_{r}^{T} v_{r}$ of the current and voltage vectors at resistors can 
be recast as $i_{r}^{T} R i_{r}$, and therefore $i_{r}^{T} v_{r}>0$ for all non-vanishing $i_{r}, v_{r}$ if and only if $R$ is a positive definite matrix. The same idea applies in terms of incremental matrices to define strictly locally passive devices; find details e.g. in [36].

Matrix pencils and semiexplicit DAEs. Given two matrices $A, B$ in $\mathbb{R}^{n \times n}$ the matrix pencil $\{A, B\}$ is defined as the one-parameter family $\lambda A+B[22,27,28,29,30]$. If the polynomial (in $\lambda$ ) $\operatorname{det}(\lambda A+B)$ does not vanish identically (that is, if there exists at least one value of $\lambda$ for which $\operatorname{det}(\lambda A+B) \neq 0$ ), the matrix pencil is called regular. The (finite) eigenvalues of a regular matrix pencil $\{A, B\}$ are the values of $\lambda \in \mathbb{C}$ for which $\operatorname{det}(\lambda A+B)=0$. The polynomial $\operatorname{det}(\lambda A+B)$ has degree $m \leq n$, with $m<n$ when $A$ is a singular matrix; in this case we say that the pencil has $n-m$ infinite eigenvalues.

A system of the form

$$
\begin{aligned}
x^{\prime} & =f(x, y) \\
0 & =g(x, y)
\end{aligned}
$$

is called a semiexplicit differential-algebraic equation (DAE) $[22,27,28,29]$. It is said to be index one if the matrix $g_{y}$ of partial derivatives of $g$ with respect to the variables $y$ is non-singular.

Given an equilibrium point $\left(x^{*}, y^{*}\right)$ of $(44)$, that is, a point where $f\left(x^{*}, y^{*}\right)=0, g\left(x^{*}, y^{*}\right)=0$, the linear stability properties of the equilibrium are defined by the matrix pencil

$$
\lambda\left(\begin{array}{ll}
I & 0 \\
0 & 0
\end{array}\right)-\left(\begin{array}{ll}
f_{x}\left(x^{*}, y^{*}\right) & f_{y}\left(x^{*}, y^{*}\right) \\
g_{x}\left(x^{*}, y^{*}\right) & g_{y}\left(x^{*}, y^{*}\right)
\end{array}\right)
$$

where the identity block in the first matrix has the dimension of $x$; cf. $[22,27]$.

\section{References}

[1] D. B. Strukov, G. S. Snider, D. R. Stewart and R. S. Williams, The missing memristor found, Nature 453 (2008) 80-83.

[2] B. C. Bao, Z. Liu and H. Leung, Is memristor a dynamic element?, Electronics Letters 49 (2013) 1523-1525.

[3] D. Biolek, Z. Biolek and V. Biolkova, SPICE modeling of memristive, memcapacitive and meminductive systems, Proc. Eur. Conf. Circuit Theor. Design 2009, pp. 249-252, 2009.

[4] F. Corinto, A. Ascoli and M. Gilli, Analysis of current-voltage characteristics for memristive elements in pattern recognition systems, Int. J. Circuit Theory Appl. 40 (2012) 1277-1320.

[5] M. Di Ventra, Y. V. Pershin and L. O. Chua, Circuit elements with memory: memristors, memcapacitors and meminductors, Proc. IEEE 97 (2009) 1717-1724.

[6] M. Itoh and L. O. Chua, Memristor oscillators, Int. J. Bifur. Chaos 18 (2008) 3183-3206. 
[7] L. Jansen, M. Matthes and C. Tischendorf, Global unique solvability for memristive circuit DAEs of index 1, Int. J. Circuit Theory Appl., in press, 2014.

[8] D. Jeltsema and A. Doria-Cerezo, Port-Hamiltonian formulation of systems with memory, Proc. IEEE 100 (2012) 1928-1937.

[9] O. Kavehei, A. Iqbal, Y. S. Kim, K. Eshraghian, S. F. Al-Sarawi and D. Abbott, The fourth element: characteristics, modelling and electromagnetic theory of the memristor, Proc. $R$. Soc. A 466 (2010) 2175-2202.

[10] M. Messias, C. Nespoli and V. A. Botta, Hopf bifurcation from lines of equilibria without parameters in memristors oscillators, Int. J. Bifur. Chaos 20 (2010) 437-450.

[11] B. Muthuswamy and L. O. Chua, Simplest chaotic circuit, Int. J. Bifur. Chaos 20 (2010) $1567-1580$.

[12] Y. V. Pershin and M. Di Ventra, Memory effects in complex materials and nanoscale systems, Advances in Physics 60 (2011) 145-227.

[13] Y. V. Pershin and M. Di Ventra, Neuromorphic, digital and quantum computation with memory circuit elements, Proc. IEEE 100 (2012) 2071-2080.

[14] R. Riaza, First order mem-circuits: modeling, nonlinear oscillations and bifurcations, IEEE Trans. Circ. Sys. I 60 (2013) 1570-1583.

[15] R. Tetzlaff and T. Schmidt, Memristors and memristive circuits - An overview, Proceedings ISCAS'2012, pp. 1590-1595, 2012.

[16] A. Ascoli, T. Schmidt, R. Tetzlaff and F. Corinto, Application of the Volterra series paradigm to memristive systems, in R. Tetzlaff (ed.), Memristors and Memristive Systems, pp. 163-191, Springer, 2014.

[17] L. O. Chua, Memristor - The missing circuit element, IEEE Trans. Circuit Theory 18 (1971) 507-519.

[18] L. O. Chua, Nonlinear circuit foundations for nanodevices, Part I: The four-element torus, Proc. IEEE 91 (2004) 1830-1859.

[19] F. Corinto, A. Ascoli and M. Gilli, Nonlinear dynamics of memristor oscillators, IEEE Trans. Circuits and Systems - I 58 (2011) 1323-1336.

[20] L. O. Chua and A. D. Deng, Impasse points, I: Numerical aspects, Int. J. Circuit Theory Appl., 17 (1989) 213-235.

[21] L. O. Chua and A. D. Deng, Impasse points, II: Analytical aspects, Int. J. Circuit Theory Appl., 17 (1989) 271-282.

[22] R. Riaza, Differential-Algebraic Systems, World Scientific, 2008. 
[23] S. S. Sastry and C. A. Desoer, Jump behavior of circuits and systems, IEEE Trans. Circuits and Systems 28 (1981) 1109-1124.

[24] L. O. Chua, C. A. Desoer and E. S. Kuh, Linear and Nonlinear Circuits, McGraw-Hill, 1987.

[25] D. P. Brown, Derivative-explicit differential equations for RLC graphs, Journal of the Franklin Institute 275 (1963) 503-514.

[26] R. Riaza, Manifolds of equilibria and bifurcations without parameters in memristive circuits, SIAM J. Appl. Math. 72 (2012) 877-896.

[27] K. E. Brenan, S. L. Campbell and L. R. Petzold, Numerical Solution of Initial-Value Problems in Differential-Algebraic Equations, SIAM, 1996.

[28] P. Kunkel and V. Mehrmann, Differential-Algebraic Equations. Analysis and Numerical Solution, EMS, 2006.

[29] R. Lamour, R. März and C. Tischendorf, Differential-Algebraic Equations: A Projector Based Analysis, Springer, 2013.

[30] F. R. Gantmacher, The Theory of Matrices, Chelsea, 1959.

[31] R. E. Beardmore, The singularity-induced bifurcation and its Kronecker normal form, SIAM J. Matrix Anal. Appl. 23 (2001) 126-137.

[32] V. Venkatasubramanian, H. Schättler and J. Zaborszky, Local bifurcations and feasibility regions in differential-algebraic systems, IEEE Tr. Aut. Contr. 40 (1995) 1992-2013.

[33] B. Bollobás, Modern Graph Theory, Springer-Verlag, 1998.

[34] R. Diestel, Graph Theory, Springer-Verlag, 2000.

[35] L. R. Foulds, Graph Theory Applications, Springer, 1992.

[36] L. O. Chua, Dynamic nonlinear networks: state-of-the-art, IEEE Trans. Circuits and Systems 27 (1980) 1059-1087. 